\title{
Site-Selective Dynamics of Azidolysozyme
}

\author{
Seyedeh Maryam Salehi and Markus Meuwly* \\ Department of Chemistry, University of Basel, Klingelbergstrasse 80 , CH-4056 Basel, \\ Switzerland. \\ E-mail: m.meuwly@unibas.ch
}




\begin{abstract}
The spectroscopic response of and structural dynamics around all azido-modified alanine residues $\left(\mathrm{AlaN}_{3}\right)$ in Lysozyme is characterized. It is found that $\mathrm{AlaN}_{3}$ is a positionally sensitive probe for the local dynamics, covering a frequency range of $\sim 15$ $\mathrm{cm}^{-1}$ for the center frequency of the line shape. This is consistent with findings from selective replacements of amino acids in PDZ2 which reported a frequency span of $\sim 10 \mathrm{~cm}^{-1}$ for replacements of Val, Ala, or Glu by azidohomoalanine (AHA). For the frequency fluctuation correlation functions (FFCFs) the long-time decay constants $\tau_{2}$ range from $\sim 1$ to $\sim 10 \mathrm{ps}$ which compares with experimentally measured correlation times of 3 ps. Attaching azide to alanine residues can yield dynamics that decays to zero on the few ps time scale (i.e. static component $\Delta_{0} \sim 0 \mathrm{ps}^{-1}$ ) or to a remaining, static contribution of $\sim 0.5 \mathrm{ps}^{-1}$ (corresponding to $2.5 \mathrm{~cm}^{-1}$ ), depending on the local environment on the $10 \mathrm{ps}$ time scale. The magnitude of the static component correlates qualitatively with the degree of hydration of the spectroscopic probe. Although attaching azide to alanine residues is found to be structurally minimally invasive with respect to the overall protein structure, analysis of the local hydrophobicity indicates that the hydration around the modification site differs for modified and unmodified alanine residues, respectively.
\end{abstract}

\title{
Introduction
}

Understanding the structural and functional dynamics of proteins in the condensed phase is a prerequisite for characterizing cellular processes at a molecular level. ${ }^{1}$ As an example, knowledge of the mechanisms and physical principles underlying protein-ligand recognition facilitates rational drug design for treatment of diseases. ${ }^{2,3}$ One possibility to directly and quantitatively probe the structure and dynamics of proteins and protein-ligand complexes is vibrational, in particular 2-dimensional infrared (2D-IR) spectroscopy. ${ }^{4}$ 
Given the spectroscopic response of proteins in solution that cover the range up to $\sim 1700$ $\mathrm{cm}^{-1}$ and frequencies above $\sim 2800 \mathrm{~cm}^{-1}$, suitable vibrational labels should absorb in the window between $\sim 1700$ and $\sim 2800 \mathrm{~cm}^{-1} .{ }^{5,6}$ A range of such probes has been proposed and considered in the past, including cyanophenylalanine,${ }^{7}$ nitrile-derivatized amino acids, ${ }^{8}$ the sulfhydryl band of cysteines, ${ }^{9}$ deuterated carbons, ${ }^{10}$ non-natural labels consisting of metaltricarbonyl modified with a $-\left(\mathrm{CH}_{2}\right)_{\mathrm{n}^{-}}$linker, ${ }^{11}$ nitrile labels, ${ }^{12}$ cyano ${ }^{13}$ and $\mathrm{SCN}^{14}$ groups, or cyanamide. ${ }^{15}$ Another promising and sensitive label that was recently used is azidohomoalanine $(\mathrm{AHA})^{16}$ for which it has been demonstrated that it can be used to characterize the recognition site between the PDZ2 domain and its binding partner to provide site-specific insight into the underlying mechanisms of how signaling proteins function. ${ }^{17}$

The noncanonical amino acid AHA absorbs around $\sim 2100 \mathrm{~cm}^{-1}$ with a comparatively large extinction coefficient of up to $400 \mathrm{M}^{-1} \mathrm{~cm}^{-1} .{ }^{16}$ From a preparative perspective attachment of $\mathrm{N}_{3}^{-}$to alanine (to give $\mathrm{AlaN}_{3}$ ) and $\mathrm{AHA}$ and incorporation at almost any position of a protein through known expression techniques has been demonstrated. ${ }^{18}$ Furthermore, attachment of an $\mathrm{N}_{3}^{-}$probe is a spatially small modification and the chemical perturbations induced are expected to be small. This makes $\mathrm{AlaN}_{3}$ and AHA worthwhile modifications to probe local protein dynamics.

Optical spectroscopy, and especially two-dimensional infrared (2D-IR) spectroscopy, quantitatively provides information about the structure and dynamics of the solvent environment surrounding a probe molecule. ${ }^{19}$ Such techniques can also be used to measure the subpicosecond to picosecond dynamics in condensed-phase systems. With that, the coupling between inter- and intramolecular degrees of freedom such as the hydrogen bonding network in solution, or structural features of biological macromolecules can be investigated by monitoring the fluctuation of fundamental vibrational frequencies of a probe molecule or ligand attached to a complex or a biological macromolecule. The possibility to use infrared spectroscopy for 
characterizing protein-ligand complexes has already been proposed for the nitrile containing inhibitor IDD743 complexed with WT and mutant human aldose reductase ${ }^{20}$ and explicitly demonstrated for cyano-benzene in the active site of WT and mutant lysozyme. ${ }^{21}$

The AHA label was previously used in 2D-IR spectroscopy studies of ligand binding to the PDZ2 domain. ${ }^{16}$ The spectral changes observed for various modified pepdidic binders were consistent with the known X-ray structure of the wild-type peptide bound to the protein. This suggests that AHA is suitable as a specific IR reporter and to highlight subtle changes of the electrostatic environment on the protein surface. ${ }^{17}$ In the present work, attaching $\mathrm{N}_{3}^{-}$ to all alanine residues in Lysozyme in succession is used to characterize the local dynamics around such modification site.

Recent investigations have demonstrated that the vibrational dynamics of $\mathrm{N}_{3}^{-}$in the gas phase and in solution can be captured quantitatively. ${ }^{22}$ Based on high-level electronic structure calculations at the multi-reference configuration interaction (MRCI) level of theory and representing the 3-dimensional potential energy surface (PES) as a reproducing kernel Hilbert space (RKHS), ${ }^{23,24}$ the infrared spectroscopy in the gas and condensed phase was correctly described. Also, the frequency correlation function exhibited time scales consistent with experiment which suggests that the coupling between solvent and solute was correctly described.

The present work explores the local dynamics of all alanine residues in lysozyme as a typical model system by attaching $\mathrm{N}_{3}^{-}$as a spectroscopic reporter. First, the computational methods are summarized. Then, the structural dynamics and spectroscopy for all $14 \mathrm{AlaN}_{3}$ labels is discussed and the local dynamics and hydration are explored. Finally, conclusions are drawn. 


\section{Methods}

\section{Molecular Dynamics Simulations}

For the Molecular Dynamics (MD) simulations of WT and modified Lysozyme in solution, $\mathrm{CHARMM}^{25}$ together with the $\mathrm{CHARMM}^{26}$ force field was used. A suitably modified version of CHARMM was employed for the simulations with the 3-dimensional RKHS PESs (see below). ${ }^{22}$ The initial lysozyme structure was the X-ray structure $\left(3 \mathrm{FE} 0{ }^{27}\right)$. Simulations of Lysozyme in TIP3P water $^{28}$ were carried out in a cubic box of size $(62.1)^{3} \AA^{3}$. Figure 1 shows the structure of the system for the present work in which $\mathrm{N}_{3}^{-}$is attached individually to each of the 14 Ala residues, replacing one hydrogen atom of the terminal $\mathrm{CH}_{3}$ group. This yields Azidoalanine-modified Lysozyme. ${ }^{18}$ Compared with protein structures in which AHA is introduced, the two modifications differ by one $\mathrm{CH}_{2}$-group. ${ }^{18}$

The systems were minimized, heated for $25 \mathrm{ps}$ and equilibrated for $100 \mathrm{ps}$ in the $N V T$ ensemble. Production runs, 2 ns in length, were carried out in the NVT ensemble, with coordinates saved every 5 fs for subsequent analysis. All nonbonded interactions were treated with a $14 \AA$ cutoff switched at $10 \AA,{ }^{29}$ and bonds involving hydrogen atoms are constrained using SHAKE. ${ }^{30}$ 


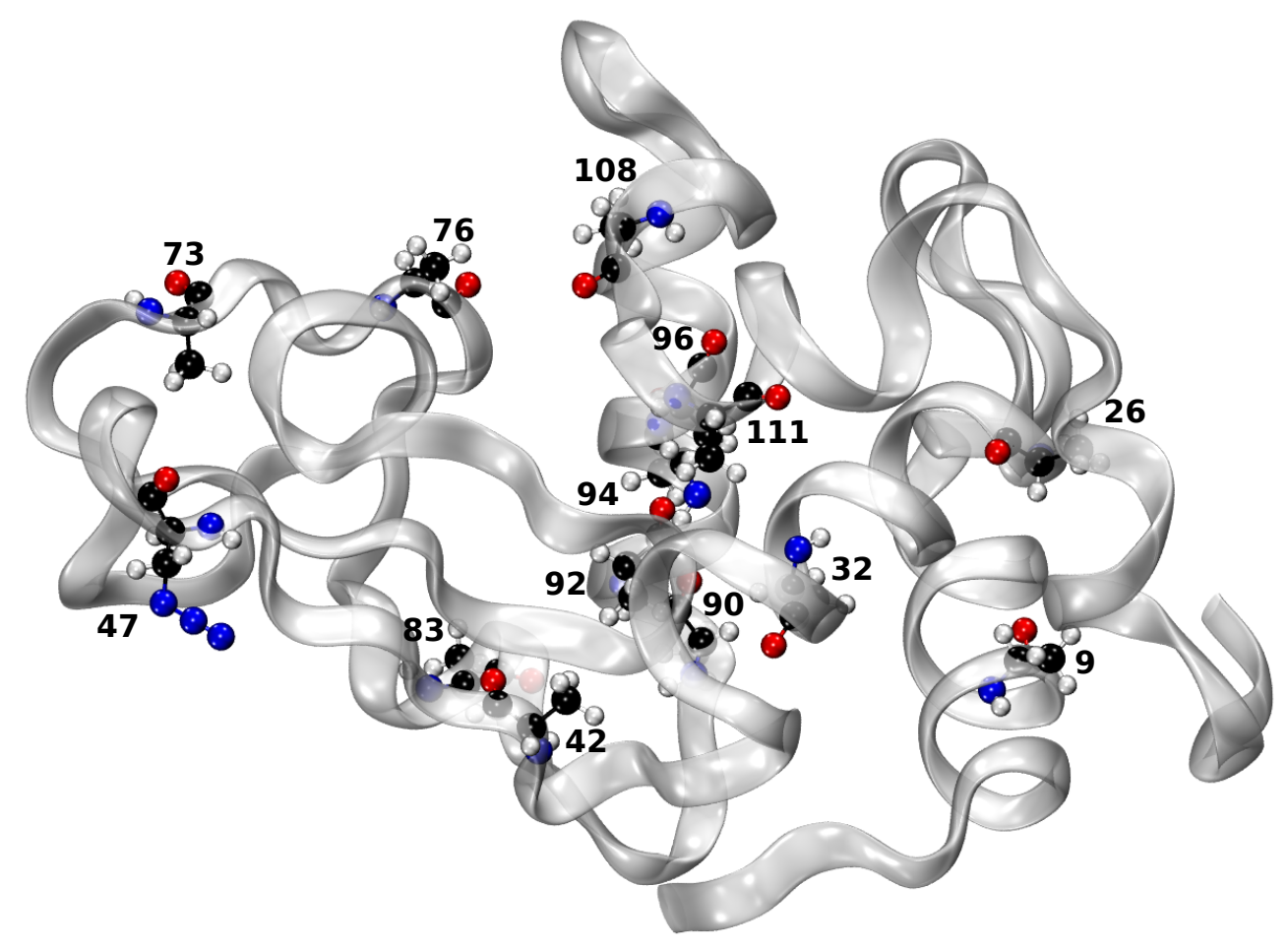

Figure 1: Structure of Lysozyme with positions of Alanine residues indicated. The Alanine residues are at positions $9,26,32,42,47,73,76,83,90,92,94,96,108,111$. Ala residues are displayed as CPK spheres and the rest of the protein structure is shown as NewRibbons. As an example, $\mathrm{AlaN}_{3}$ is shown at residue 47.

\section{Energy Function for the Spectroscopic Probe}

For representing the 3-dimensional energy function of the $\mathrm{N}_{3}^{-}$label two strategies were pursued. First, the existing 3-dimensional PES for $\mathrm{N}_{3}^{-}$, computed at the MRCI+Q level of theory in the gas phase, was used to describe the stretching and bending distortions of the label attached to the $\mathrm{CH}_{2}$ group of alanine.

Because the $\mathrm{N}_{3}^{-}$moiety and the rest of the Ala residue are not fully electronically decoupled, a second approach was pursued. For this, the structure of AHA was optimized at the MP2/aug-cc-pVTZ level of theory. Next, the structure of AHA was frozen except for the coordinates involving the spectroscopic label. Then, a new 3-dimensional PES was computed at the pair natural orbital based coupled cluster level (PNO-LCCSD(T)-F12) ${ }^{31,32}$ together 
with the aug-cc-pVTZ basis set ${ }^{33}$ using the MOLPRO suite of codes. ${ }^{34}$ As for the gas phase $\mathrm{PES},{ }^{22}$ the $a b$ initio energies were calculated in Jacobi coordinates $(R, r, \theta)$, see Figure $2 \mathrm{~B}$, where $r$ is the distance between the nitrogen atoms N1 and N2, $R$ is the distance between their center of mass and the atom N3, and $\theta$ is the angle between $\vec{r}$ and $\vec{R}$. The angular grid $(\theta)$ used here contains 5 Gauss-Legendre quadrature points between $156^{\circ}$ and $180^{\circ}$. The radial grids include 16 points along $r$ ranging from 0.90 to $1.51 \AA$ and 16 points along $R$ between 1.45 and $2.12 \AA$. The PNO-LCCSD(T)-F12 level of theory was chosen as it combines accuracy with feasibility for the present problem because recomputing the MRCI PES for AHA is computationally intractable.

For both PESs the parameters for the C-N3 stretch, the C-C-N3 and the C-N3-N2 bend are those from Swissparam. ${ }^{35}$ All remaining parameters for the alanine residues were those of the CHARMM force field and were not readjusted after attaching $\mathrm{N}_{3}^{-}$to guarantee compatibility with the CHARMM22 force field.

To carry out MD simulations for labelled Lysozyme, a continuous and differentiable representation of the ab initio energies is required. For this, a reproducing kernel Hilbert spacebased representation ${ }^{23,24}$ is used. A RKHS representation provides approximate values for a function $f(x)$ at positions $x$, away from the grid points $x_{i}$. For this, the linear problem $f\left(x_{i}\right)=\sum_{j} \alpha_{j} k\left(x_{i}, x_{j}\right)$ for the 1-dimensional kernels is solved which yields the coefficients $\alpha_{j}$. There are many possible choices for the kernel functions $k(\cdot, \cdot)$ but inverse powers of the distance have been found to perform well for intermolecular interactions. ${ }^{23,36,37}$ For multidimensional problems, tensor products of 1-dimensional kernels can be used. ${ }^{24,38}$

For the present work, the 3-dimensional kernel $K$ is

$$
K\left(X, X^{\prime}\right)=k^{(n, m)}\left(R, R^{\prime}\right) k^{(n, m)}\left(r, r^{\prime}\right) k^{(2)}\left(z, z^{\prime}\right)
$$


where $X$ stands for all dimensions involved, $r$, and $R$ are as defined above (see also Figure $2)$, and $z=\frac{1-\cos (\theta)}{2}$ maps the angle $\theta$ onto the interval $[0,1]$. Reciprocal power decay kernels $\left(k^{(n, m)}\right)$ with smoothness $n=2$ and asymptotic decay $m=6$

$$
k^{(2,6)}\left(x, x^{\prime}\right)=\frac{1}{14} \frac{1}{x_{>}^{7}}-\frac{1}{18} \frac{x_{<}}{x_{>}^{8}},
$$

are used for $r$ and $R$ whereby $x_{>}$and $x_{<}$are the larger and smaller values of $x$ and $x^{\prime}$, respectively. For the angular degree of freedom, a Taylor spline kernel

$$
k^{(2)}\left(z, z^{\prime}\right)=1+z_{<} z_{>}+2 z_{<}^{2} z_{>}-\frac{2}{3} z_{<}^{3}
$$

is used.

Charges were calculated for the optimized structure of $\mathrm{AlaN}_{3}$ at the MP2/aug-cc-pVTZ level of theory from an $\mathrm{NBO}^{39}$ analysis using Gaussian ${ }^{40}$ and scaled to maintain overall neutrality. This yields a charge of -0.2460 e for the nitrogen atom N1 attached to $\mathrm{CH}_{2}$ group, 0.1607e for the central N2 and $-0.0464 e$ for the terminal nitrogen N3.

\section{Frequency Fluctuation Correlation Function and Lineshape}

From each production simulation, $4 \times 10^{5}$ snapshots are taken as a time-ordered series for computing the frequency fluctuation correlation function (FFCF) $\langle\delta \omega(0) \delta \omega(t)\rangle$ and line shapes. Here, $\delta \omega(t)=\omega(t)-\langle\omega(t)\rangle$ and $\langle\omega(t)\rangle$ is the ensemble average of the transition frequency. The FFCF was determined from instantaneous harmonic vibrational frequencies based on a normal mode analysis. ${ }^{41}$ Normal modes were determined for each snapshot after minimizing the structure of the $\mathrm{N}_{3}$ label and keeping the surrounding solvent frozen. Thus, frequency trajectories $\omega_{i}(t)$ for label $i$ were obtained for the asymmetric stretch vibration of $\mathrm{N}_{3}$ attached 
to Ala. From the FFCF the line shape function

$$
g(t)=\int_{0}^{t} \int_{0}^{\tau^{\prime}}\left\langle\delta \omega\left(\tau^{\prime \prime}\right) \delta \omega(0)\right\rangle d \tau^{\prime \prime} d \tau^{\prime}
$$

is determined within the cumulant approximation. To compute $g(t)$, the FFCF is numerically integrated using the trapezoidal rule and the 1D-IR spectra is then calculated as ${ }^{42}$

$$
I(\omega)=2 \Re \int_{0}^{\infty} e^{i(\omega-\langle\omega\rangle) t} e^{-g(t)} e^{-\frac{t}{2 T_{1}}} e^{-2 D_{\mathrm{OR}} t} d t,
$$

where $\langle\omega\rangle$ is the average transition frequency obtained from the distribution, $T_{1}(0.8 \pm 0.1 \mathrm{ps})$ is the vibrational relaxation time and $D_{\mathrm{OR}}=1 / 6 T_{R}$ with $T_{R}=1.3 \pm 0.3 \mathrm{ps}$ is the rotational diffusion coefficient which accounts for lifetime broadening. ${ }^{43}$

From the FFCF, the decay time can be determined by fitting the FFCF to a general expres$\operatorname{sion}^{44}$

$$
\langle\delta \omega(t) \delta \omega(0)\rangle=a_{1} \cos (\gamma t) e^{-t / \tau_{1}}+\sum_{i=2}^{n} a_{i} e^{-t / \tau_{i}}+\Delta_{0}
$$

where $a_{i}, \tau_{i}, \gamma$ and $\Delta_{0}$ are fitting parameters. The decay times $\tau_{i}$ of the frequency fluctuation correlation function reflect the characteristic time-scales of the solvent fluctuations to which the solute degrees of freedom are coupled. In all cases the FFCFs were fitted to an expression containing two decay times (i.e. $n_{\max }=2$ ) using an automated curve fitting tool from the SciPy library. ${ }^{45}$ 


\section{Results}

\section{The Potential Energy Surface for the $\mathrm{N}_{3}^{-}$Label}

Two PESs for the energetics of the $\mathrm{N}_{3}^{-}$are considered in the present work. One is based on earlier MRCI+Q calculations with the aug-cc-pVTZ basis set for $\mathrm{N}_{3}^{-}$in the gas phase ${ }^{22}$ which was used without change for the simulation of the $\mathrm{AlaN}_{3}$ unit. The second one was the LCCSD(T) PES for AHA which included coupling between the $\mathrm{N}_{3}^{-}$probe and the amino acid framework. The RKHS representations of the two PESs are reported in Figures 2A and $\mathrm{B}$ and the scans within CHARMM are shown in panels $\mathrm{C}$ and $\mathrm{D}$.

The RKHS representation of the PES for AHA was constructed from 1280 ab initio LCCSD(T)F12 energies. An additional 230 ab initio energies are calculated at off-grid geometries to assess the quality of the RKHS representation. Figure S1 shows the correlation between the reference energies and the RKHS with a correlation coefficient of $R^{2}=0.9999$ and the root mean squared error is $0.38 \mathrm{kcal} / \mathrm{mol}$. This confirms the high quality of the RKHS PES.

Figure $2 \mathrm{~A}$ and $\mathrm{B}$ report the RKHS interpolation of the ab initio calculated energies whereas Figures $2 \mathrm{C}$ and $\mathrm{D}$ are from scanning the $r$ and $R$ coordinates for AHA in the gas phase in CHARMM. Comparing the PNO-LCCSD(T)-F12 PES (Figure 2A) with that at the MRCI+Q level of theory (Figure 2B) shows that the minima for the two are slightly displaced $(r=1.19 \AA$ vs. $r=1.24 \AA$ and $R=1.77 \AA$ vs. $R=1.76 \AA)$. Furthermore, the LCCSD(T) PES is steeper along both, the $r$ and $R$ coordinates, which pushes the respective vibrations up compared with the MRCI+Q PES, see Figure S2. Differences between the two PESs are due to both, the methods (MRCI+Q vs. PNO-LCCSD(T)-F12) and the model system ( $\mathrm{N}_{3}^{-}$vs. AHA) considered. Comparing the isolated, gas-phase PESs (panels A and B) with those for $\mathrm{AlaN}_{3}$ (panels $\mathrm{C}$ and D) indicates that the PESs are close but not identical due to coupling between the spectroscopic probe and the alanine residue. 

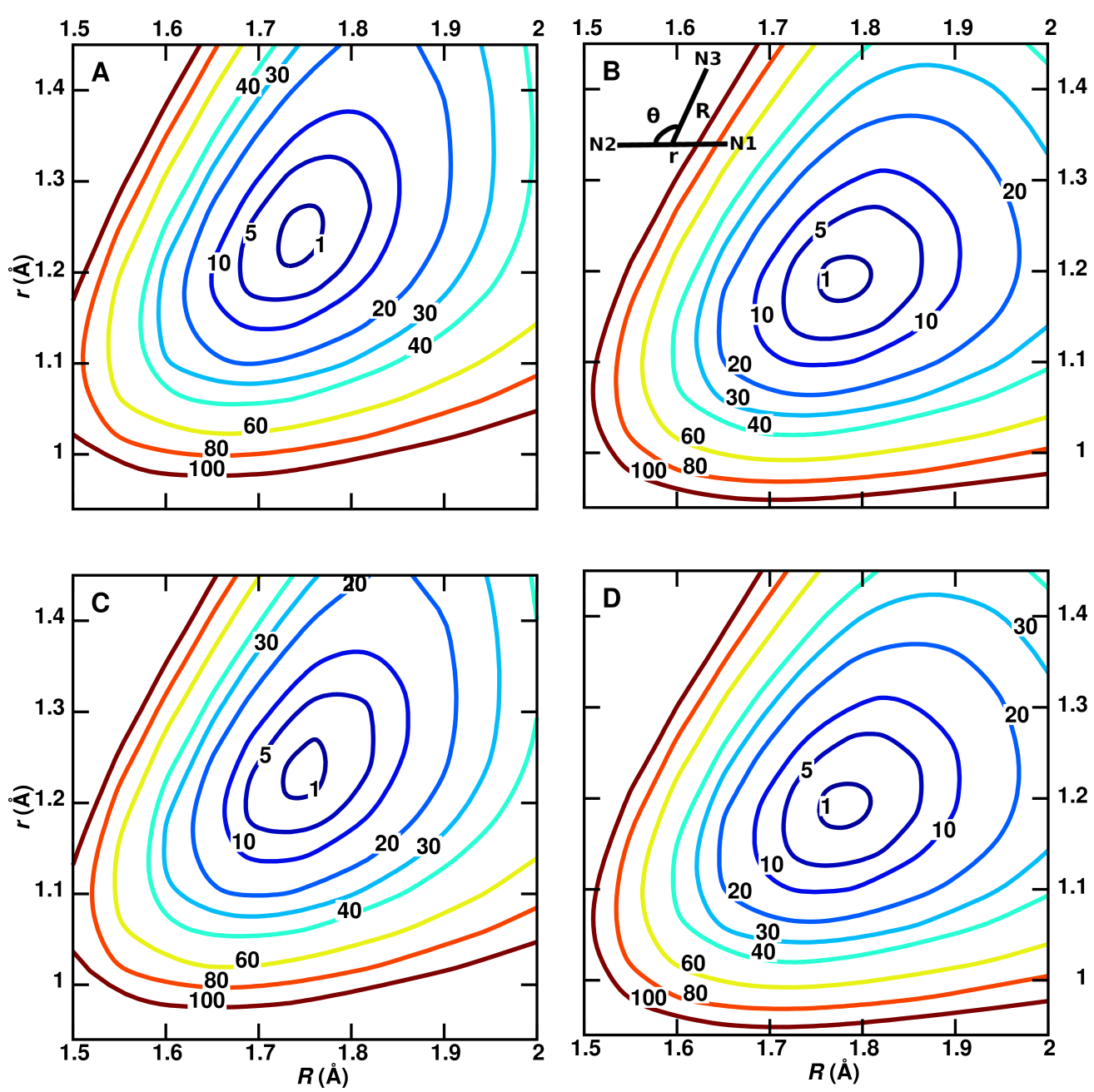

Figure 2: Contour diagrams of the RKHS representations for AHA (panel A, PNOLCCSD(T)-F12) and $\mathrm{N}_{3}^{-}$(panel B, MRCI+Q/aug-cc-pVQZ) PESs based on ab initio points calculated in Jacobi coordinates $(R, r, \theta)$ for $\theta=180^{\circ}$, see inset in panel B. Panels $\mathrm{C}$ and $\mathrm{D}$ report the corresponding CHARMM energies for AHA. All energies are in $\mathrm{kcal} / \mathrm{mol}$ and relative to the zero of energy which is the minimum energy structure.

In the following, all MD simulations were carried out with the PNO-LCCSD(T)-F12 PES as it yields harmonic frequencies for $\mathrm{AlaN}_{3}$ around $2110 \mathrm{~cm}^{-1} \mathrm{~cm}^{-1}$ (see Table 1) which is consistent with those experimentally observed for the replacement of $\mathrm{AHA}^{17}$ in PDZ2 domain at $2114 \mathrm{~cm}^{-1}$ and for AlaN3 ${ }^{46}$ in $\mathrm{H}_{2} \mathrm{O}$ at $2116 \mathrm{~cm}^{-1}$, respectively. Moreover, the influence of the covalent bonding to the Alanine residue is included in the construction of the potential energy surface. Additional refinements of the PES would, in principle, be possible through 
morphing ${ }^{47,48}$ but were not deemed necessary for the present work which is mainly concerned with the differential dynamics, i.e. the relative positional sensitivity, and spectroscopy for the same label at different positions along the polypeptide chain.

\section{Structural Dynamics}

For the structural dynamics first the root mean squared deviation (RMSD) of unmodified and modified Lysozyme in solution compared with the starting X-ray structure as the reference is analyzed. For this, the RMSD of all $\mathrm{C}_{\alpha}$ atoms was considered. Figure 3 shows the RMSD for all $\mathrm{C}_{\alpha}$ atoms (blue) and those for the 14 Alanine residues (red) specifically from the 2 ns simulation of the modified protein at position Ala47. For the WT protein similar RMSD values are reported in Figure S3. The RMSD values fluctuate below or around $1 \AA$ which is indicative of a stable simulation. This suggests that attaching a $\mathrm{N}_{3}^{-}$label to Ala has an insignificant effect on the structural dynamics of Lysozyme, consistent with earlier findings for the PDZ domain for which also a minimally invasive effect was reported. ${ }^{17}$

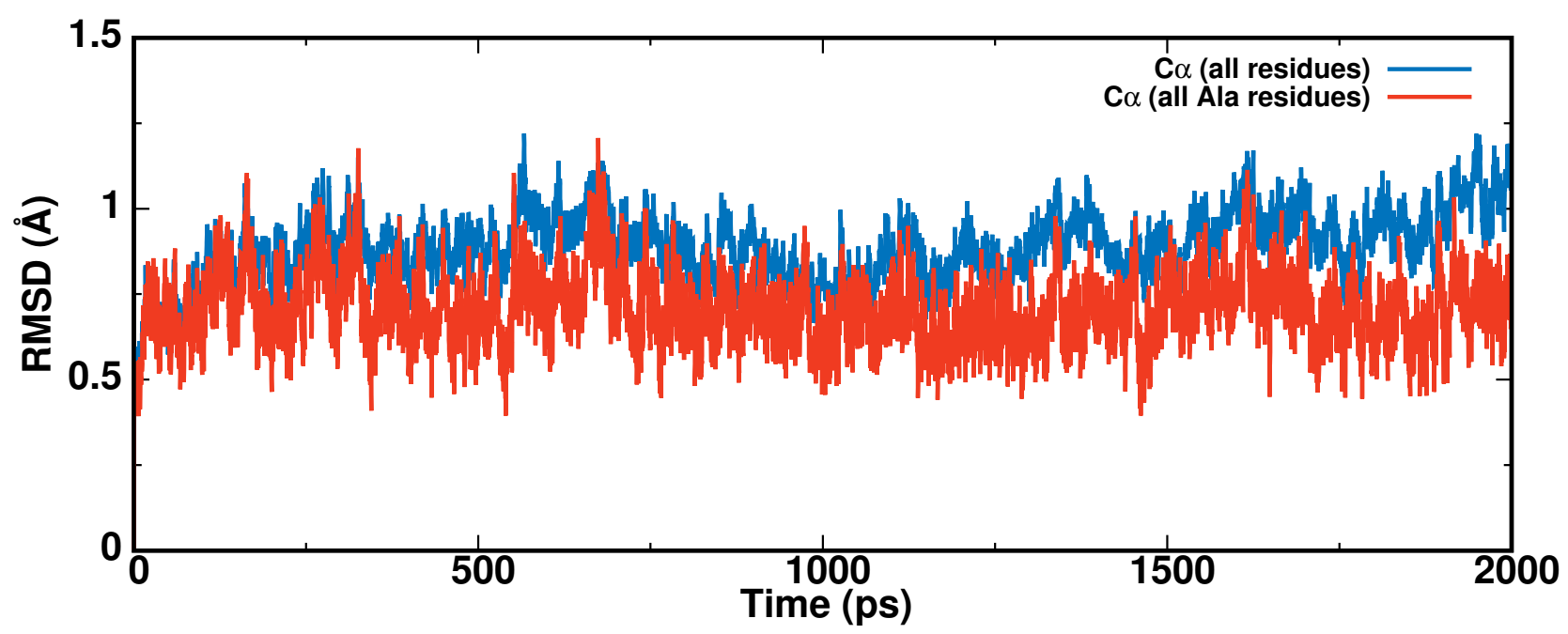

Figure 3: The structural RMSD for the $\mathrm{C}_{\alpha}$ atoms from all residues (blue) and for the 14 Ala residues (red) specifically for $\mathrm{Ala}_{3} 6 \mathrm{~N}_{3}$. 


\section{Vibrational Spectra and Frequency Correlation Functions}

First, the power spectra and frequency trajectories for the asymmetric stretch of the azide label attached to all 14 alanine residues are presented. The power spectra as determined from the Fourier transform of the N2-N3 distance correlation function are shown in Figure $4 \mathrm{~A}$ for all $\mathrm{AlaN}_{3}$ from $2 \mathrm{~ns}$ production runs. The peak maxima $\omega_{\max }$ cover a range of $\sim 20$ $\mathrm{cm}^{-1}$ (between 2160 and $2180 \mathrm{~cm}^{-1}$ ) and the full widths at half maximum (fwhm) of the spectra are around $20 \mathrm{~cm}^{-1}$. Hence, although the same energy function was used for all modified $\mathrm{AlaN}_{3}$ moieties, their power spectra differ depending on the position of the modified Ala residue along the polypeptide chain.

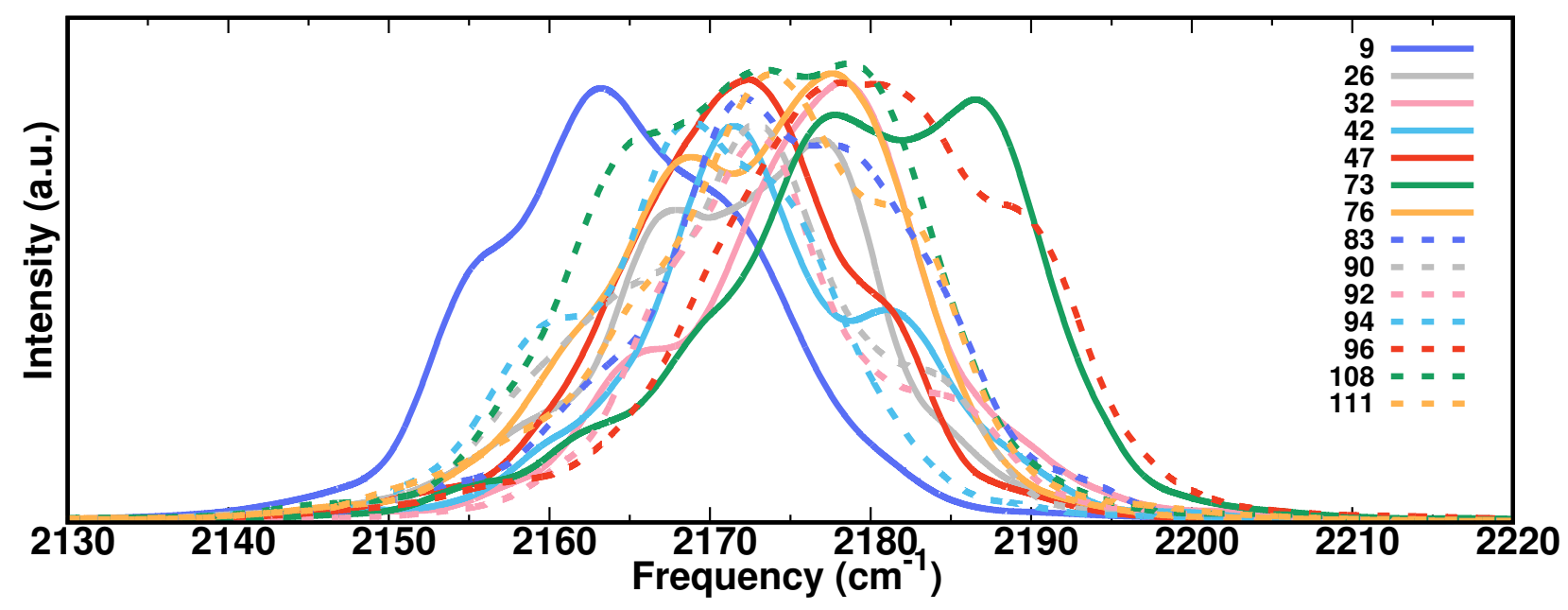

Figure 4: Power spectrum based on the $\mathrm{N} 2-\mathrm{N} 3$ separation for all modified $\mathrm{AlaN}_{3}$ residues. The position of the frequency maxima differ for most of the $\mathrm{AlaN}_{3}$ labels and cover a range between 2160 and $2180 \mathrm{~cm}^{-1}$.

The power spectra reported in Figure 4 are also representative of the infrared spectrum as shown in Figure S4. The top panel of Figure S4 reports the power spectrum and peak positions of all three modes for Ala $47 \mathrm{~N}_{3}$ with the asymmetric stretch centered around 2170 $\mathrm{cm}^{-1}$, the symmetric stretch at $1333 \mathrm{~cm}^{-1}$ and the bending mode at $610 \mathrm{~cm}^{-1}$. The bottom panel of Figure S4 demonstrates that the infrared spectrum (IR) determined from the 
dipole autocorrelation function supports the peak positions found from the power spectrum to within $2 \mathrm{~cm}^{-1}$.

Next, the frequency trajectories $\omega_{i}(t)$ for each of the spectroscopic probes $i$ from $4 \times 10^{5}$ snapshots were determined from instantaneous normal mode calculations. From the frequency time series the frequency fluctuation correlation functions (FFCFs) are obtained. They contain valuable information about the environmental dynamics around each site $i$, i.e. the azide probes of the various Ala residues considered.

The FFCFs, shown in Figure 5, are fitted to Eq. 6 with a parametrization motivated by the overall shape of the FFCF. ${ }^{44}$ This functional form has also been used in previous work. ${ }^{44,49,50}$ It is an extension of the typical multiexponential decay, which is traditionally employed ${ }^{51}$ to capture an anticorrelation at short times $(t<1 \mathrm{ps})$. Figure 5 provides a comparison between the raw data (black) and the fits (red) and Table 1 reports the corresponding fitting parameters. 


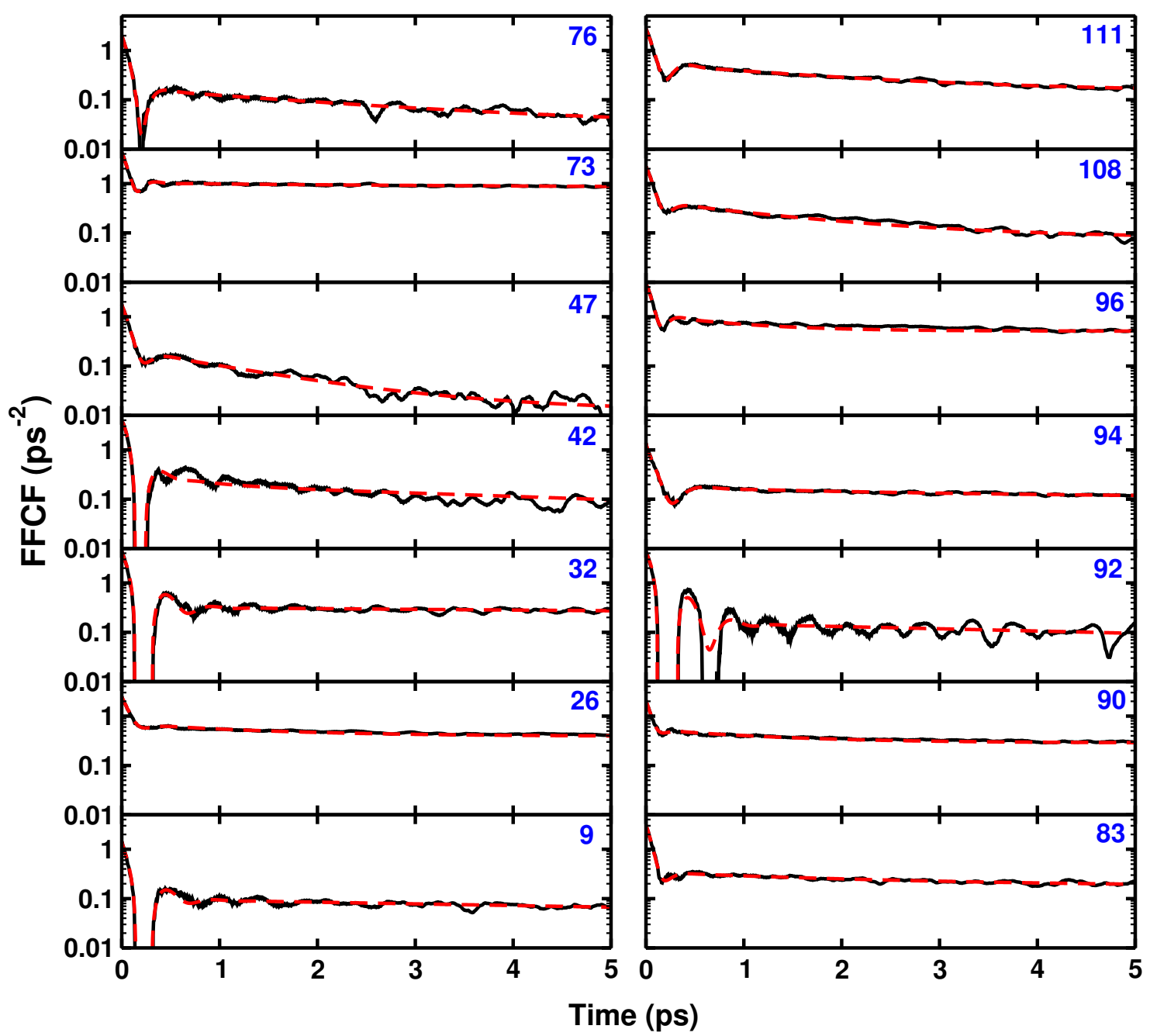

Figure 5: FFCFs from correlating the instantaneous harmonic frequencies for all $14 \mathrm{AlaN}_{3}$ in Lysozyme. The labels in each panel refer to the alanine residue which carries the azide label. Black traces are the raw data and red dashed lines the fits to Eq. 6. The $y$-axis is logarithmic.

The shape of the FFCFs can differ appreciably. Some of them display a pronounced minimum at short correlation times $(t \sim 0.1 \mathrm{ps})$ whereas others do not. This feature has also been found in previous simulations ${ }^{49}$ and has been related to the strength of the interaction between the infrared probe and its environment. ${ }^{44,50,52}$ Several of the FFCFs show one (Ala9, Ala32, Ala42, Ala76, Ala94, Ala96, Ala108, Ala111) or even two (Ala92) recurrences at short correlation times. For the remaining Alanine residues this feature is less pronounced (Ala47, Ala73, Ala83, Ala90) or entirely absent (Ala26). Similarly, some of the FFCFs exhibit clear static components $\Delta_{0} \simeq 0.5 \mathrm{ps}^{-2}$ (Ala26, Ala73, Ala96) whereas the remaining ones decay 
to zero on the $\sim 10 \mathrm{ps}$ time scale. With respect to the correlation times, the fast correlation is generally $\tau_{1} \sim 0.1 \mathrm{ps}$ whereas the long time scale ranges from $\tau_{2}=1.1$ ps to $\tau_{2}<13$ ps, see Table 1. Typically, the amplitude of the fast decay is one order of magnitude larger than that of the slow contribution (Table 1). Hence, the characteristics of the FFCFs vary considerably depending on the position at which the Alanine residue is located along the polypeptide chain. This suggests that $\mathrm{AlaN}_{3}$ is a positionally sensitive probe to provide quantitative information about the local dynamics of a protein.

Table 1: Parameters obtained from fitting the FFCF to Eq. 6 for INM frequencies for all different $\mathrm{AlaN}_{3}$ residues in lysozyme. Average frequency $\langle\omega\rangle$ of the asymmetric stretch in $\mathbf{c m}^{-1}$, the amplitudes $a_{1}$ to $a_{3}$ in $\mathbf{p s}^{-2}$, the decay times $\tau_{1}$ to $\tau_{3}$ in $\mathbf{p s}$, the parameter $\gamma$ in $\mathbf{p s}^{-1}$, the offset $\Delta_{0}$ in $\mathbf{p s}^{-2}$, and the conformationally averaged local hydrophobicity $(\mathrm{LH})$.

\begin{tabular}{r|c|rrc|cc|c||c}
\hline \hline Res & $\langle\omega\rangle$ & $a_{1}$ & $\gamma$ & $\tau_{1}$ & $a_{2}$ & $\tau_{2}$ & $\Delta_{0}$ & LH \\
\hline $\mathbf{9}$ & 2103.7 & 1.17 & 13.16 & 0.129 & 0.07 & 7.62 & 0.02 & 0.33 \\
\hline $\mathbf{2 6}$ & 2107.9 & 1.82 & 8.69 & 0.079 & 0.24 & 1.73 & 0.41 & 1.91 \\
\hline $\mathbf{3 2}$ & 2112.8 & 3.60 & 13.39 & 0.164 & 0.13 & 13.05 & 0.19 & 0.11 \\
\hline $\mathbf{4 2}$ & 2111.4 & 3.57 & 14.08 & 0.104 & 0.32 & 2.15 & 0.04 & 0.95 \\
\hline $\mathbf{4 7}$ & 2107.5 & 1.54 & 9.99 & 0.081 & 0.21 & 1.17 & 0.01 & 1.21 \\
\hline $\mathbf{7 3}$ & 2114.6 & 3.18 & 15.07 & 0.080 & 0.24 & 3.61 & 0.81 & 1.34 \\
\hline $\mathbf{7 6}$ & 2107.1 & 1.94 & 11.94 & 0.084 & 0.15 & 2.77 & 0.02 & 0.40 \\
\hline $\mathbf{8 3}$ & 2109.2 & 2.75 & 11.48 & 0.069 & 0.17 & 2.11 & 0.18 & 1.59 \\
\hline $\mathbf{9 0}$ & 2107.5 & 1.57 & 11.71 & 0.056 & 0.22 & 1.42 & 0.30 & 1.13 \\
\hline $\mathbf{9 2}$ & 2110.4 & 4.08 & 14.02 & 0.184 & 0.10 & 1.33 & 0.10 & 0.58 \\
\hline $\mathbf{9 4}$ & 2104.8 & 1.05 & 9.36 & 0.100 & 0.08 & 5.89 & 0.09 & 1.17 \\
\hline $\mathbf{9 6}$ & 2116.3 & 3.74 & 13.33 & 0.077 & 0.43 & 2.19 & 0.48 & 0.44 \\
\hline $\mathbf{1 0 8}$ & 2108.4 & 1.83 & 10.77 & 0.084 & 0.35 & 2.43 & 0.04 & 0.54 \\
\hline $\mathbf{1 1 1}$ & 2110.6 & 2.22 & 13.13 & 0.090 & 0.41 & 2.27 & 0.12 & 1.50 \\
\hline \hline
\end{tabular}

Numerical integration of $g(t)$ and using Eq. 6 yields the 1-dimensional IR spectra for each label based on instantaneous normal modes, see Figure 6. Similar to the power spectra, the center frequencies cover a range of $\sim 15 \mathrm{~cm}^{-1}$, with center frequencies of $2104 \mathrm{~cm}^{-1}$ for Ala9N 3 and $2116 \mathrm{~cm}^{-1}$ for $\mathrm{Ala}_{3} \mathrm{~N}_{3}$, and the fwhm ranges from 13 to $21 \mathrm{~cm}^{-1}$. Also, the $\omega_{\max }$ for $\mathrm{Ala}_{3} \mathrm{~N}_{3}$ (blue solid line in Figures 4 and 6) is lowest in frequency and those for Ala96N 3 (dashed red) and Ala73N 3 (solid green) are highest from the power spectra and the INM lineshapes, respectively. The blue shift of the power spectra compared with those 
from INM for the symmetric and asymmetric stretch modes was already found for $\mathrm{N}_{3}^{-}$in solution. ${ }^{22}$ The magnitude of this shift is larger in the present case probably due to coupling between the spectroscopy probe and the amino acid it is attached to.

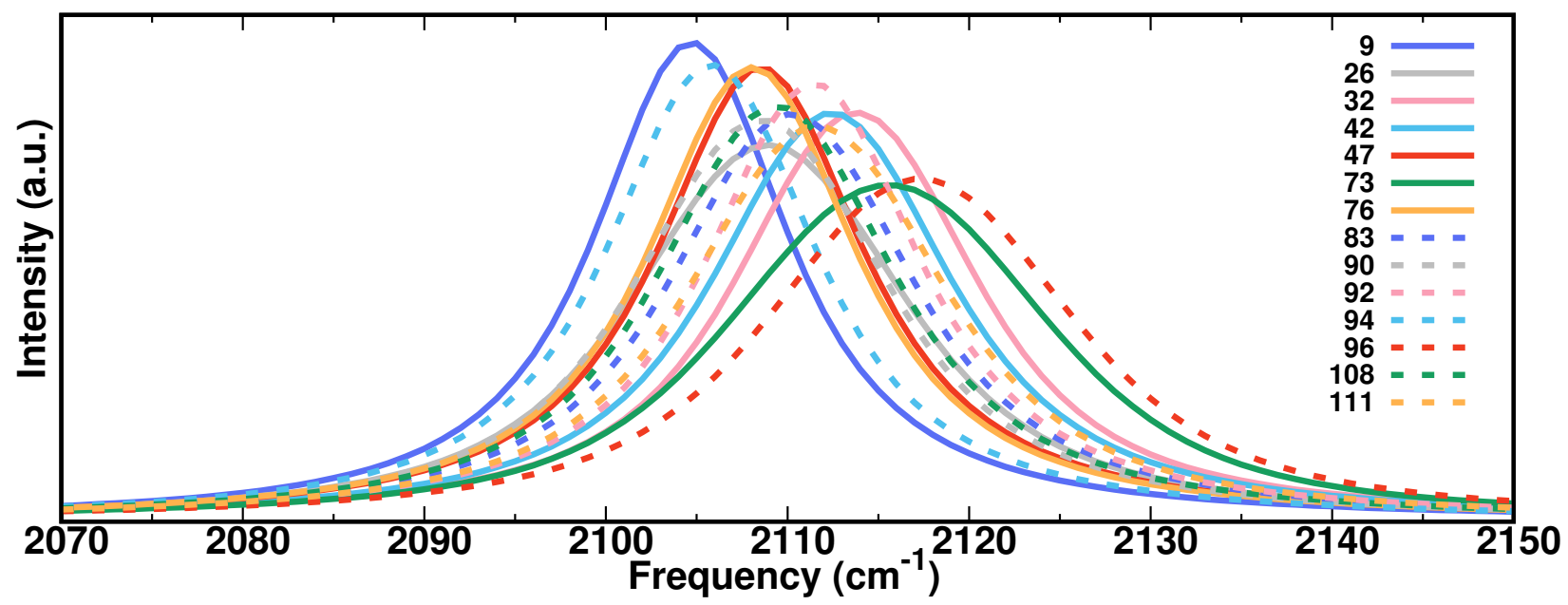

Figure 6: 1D IR spectra for all $14 \mathrm{AlaN}_{3}$ residues in Lysozyme. For the IR lineshape the raw FFCF from the INM analysis was numerically integrated to give $g(t)$ from which the 1D lineshape is obtained.

An alternative to instantaneous normal modes is to obtain instantaneous frequencies from solving the 1- or 3-dimensional nuclear Schrödinger equation. For this, the corresponding 1or 3-d PES is scanned for a given snapshot with frozen environment ${ }^{41,50,53}$ and represented as a RKHS. This is a computationally much more demanding approach, in particular in 3 spatial dimensions. ${ }^{22}$ Here, the 1-dimensional PES along the asymmetric stretch motion was mapped out for $4 \times 10^{5}$ snapshots and the nuclear Schrödinger equation was solved. Then, the FFCF was again determined and fit to Eq. 6, see Figure S5. From this, the 1-dimensional IR lineshape was determined, see solid lines in Figure S6. This was done for Ala90N 3 and Ala94N $\mathrm{N}_{3}$. As was found for the 1-d lineshapes from INM, the frequency maximum for Ala90N 3 is shifted to the blue relative to Ala94N 3 but the shift is smaller (1 $\mathrm{cm}^{-1}$ vs. $\left.3 \mathrm{~cm}^{-1}\right)$. 
Recently, the "INM", "scan" and "map" approaches have been compared for insulin monomer and dimer. ${ }^{41}$ It was found that the "INM" and "scan" approaches yield comparable 1-d infrared spectra for the amide-I bands and conclusions drawn from the spectra concerning monomeric and dimeric insulin are consistent. Nevertheless, the two approaches can differ in the absolute frequencies as is also found in the present case.

Typically, spectroscopic work has used AHA as an infrared label instead of azidoalanine as used here. To quantify the difference between $\mathrm{AlaN}_{3}$ and AHA, residue Ala47 has also been replaced by AHA through inserting an additional $\mathrm{CH}_{2}$ group before the $\mathrm{N}_{3}^{-}$label. The parametrization of the $\mathrm{CH}_{2}$ group is identical to that already used for alanine. Then, a 2 ns simulation for AHA in water was carried out and the IR spectrum was determined from an INM analysis, see Figure S7. It is found that the position of the frequency maximum for the asymmetric stretch of the azide label differs by less than $1 \mathrm{~cm}^{-1}$ from that with $\mathrm{AlaN}_{3}$ which confirms that for IR spectroscopy, the two systems are very similar.

\section{Solvent Structure and Dynamics}

Next, the solvent structuring around the modification sites is characterized. This also provides the information for an attempt to relate the spectral signatures (position of the frequency maximum, characteristics of the FFCFs) for the azide labels at different positions along the polypeptide chain with structural features and environmental properties. For this, the solvent structure around each of the $14 \mathrm{AlaN}_{3}$ probes was analyzed. First, the radial distribution functions $g(r)$ were computed along all production simulations for the 14 modified proteins, see Figure 7. The distance analyzed was the separation between the water-oxygen atom $\left(\mathrm{O}_{\mathrm{W}}\right)$ and the middle nitrogen $(\mathrm{N} 2)$ of the $\mathrm{N}_{3}$ probe in $\mathrm{AlaN}_{3}$. The corresponding 
running coordination number $N(r)$ is

$$
N(r)=4 \pi \int_{0}^{r} r^{2} g(r) \rho d r
$$

where $\rho$ is the pure water density (Figure 7B). As is shown in Figure 7, the $g(r)$ and $N(r)$ differ for the 14 modification sites.

For some of the residues (Ala26, Ala42, Ala47, Ala73, Ala76, Ala108, Ala111; Set1) the $g(r)$ exhibits a pronounced first maximum at $3.5 \leq r_{\max 1} \leq 4 \AA$ whereas for the remaining labels (Ala9, Ala32, Ala83, Ala90, Ala92, Ala94, Ala96; Set2) such a first maximum is largely absent. This suggests that the residues in Set1 are solvent exposed whereas those in Set2 are not. The total number $N(r)$ of water molecules within a distance $r$ supports this, see Figure 7B. Up to a distance of $5 \AA$, which is typically the extent of the first hydration shell, residues in Set1 contain 10 or more water molecules whereas those belonging to Set2 have not more than 1 water molecule in their vicinity.
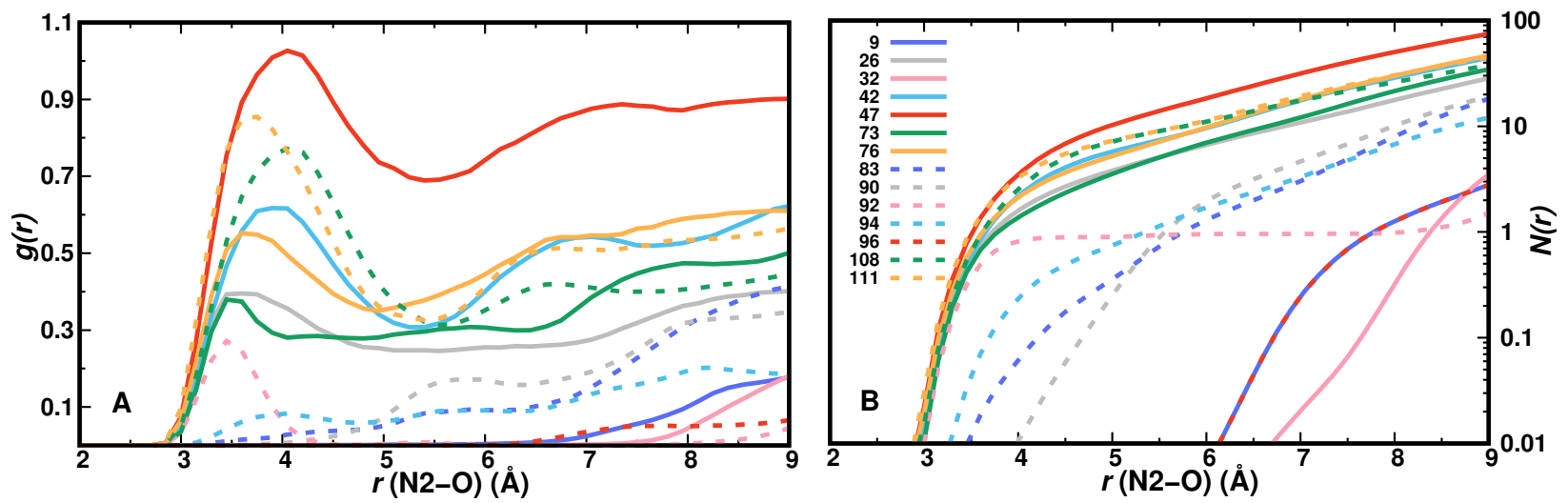

Figure 7: The radial distribution function $g(r)$ (panel A) and the number of water oxygen atoms $N(r)$ (panel B) between $\mathrm{O}$ of water and $\mathrm{N} 2$ of $\mathrm{AlaN}_{3}$ for all alanine residues from the 2 ns production simulations. The color code for the lines is given in panel B.

A structural illustration for this observation is given in Figure 8 which reports all water molecules within $7 \AA$ of Ala47N 3 (belonging to Set1) and Ala96N 3 (belonging to Set2). Con- 
sistent with Figure 7 only 3 water molecules are within the cutoff radius of atom N2 of Ala96 $\mathrm{N}_{3}$ whereas the hydration shell of Ala47N 3 is extensive.
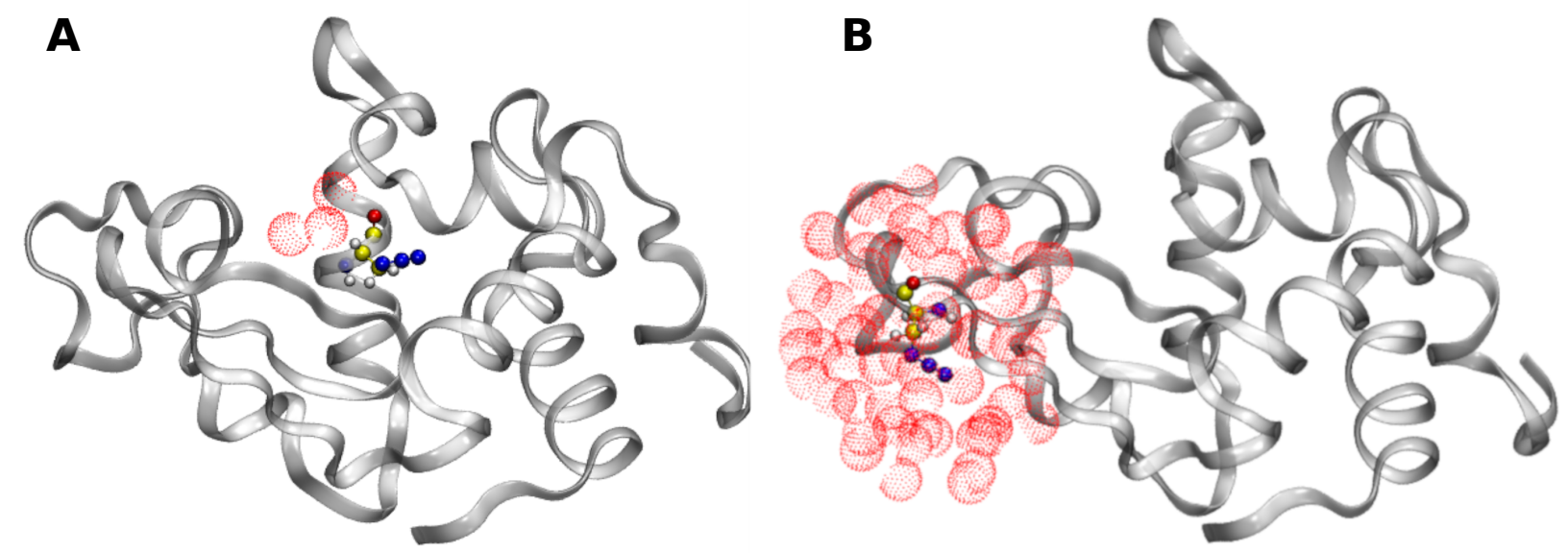

Figure 8: Solvent distribution based on the water-oxygen atoms within $7 \AA$ of any atom of residues Ala96 $\mathrm{N}_{3}$ (panel A) and $\mathrm{Ala}_{47 \mathrm{~N}_{3}}$ (panel B). The small and large hydration spheres are consistent with the $g(r)$ and $N(r)$ reported in Figure 7.

Another measure to quantify the solvent exposure of amino acids is to determine the time dependent quantity, $\delta \lambda_{\text {phob }}^{(r)}(t)$, which is referred to as the local hydrophobicity (LH) of residue $r$ at time $t .{ }^{54,55}$ This measure is based on analyzing the occupation and orientational statistics of surface water molecules at the protein/water interface, given by the three dimensional vector $\vec{\kappa}=\left(a, \cos \theta_{\mathrm{OH} 1}, \cos \theta_{\mathrm{OH} 2}\right)$. Here, $a$ is the distance of the water oxygen atom to the nearest atom of residue $r,{ }^{56}$ and $\theta_{\mathrm{OH} 1}$ and $\theta_{\mathrm{OH} 2}$ are the angles between the water $\mathrm{OH} 1$ and $\mathrm{OH} 2$ bonds and the interface normal. More specifically, the local hydrophobicity (LH) is $\delta \lambda_{\text {phob }}^{(r)}(t)=\lambda_{\text {phob }}^{(r)}(t)-\left\langle\lambda_{\text {phob }}\right\rangle_{0}$, where

$$
\lambda_{\text {phob }}^{(r)}(t)=-\frac{1}{\sum_{a=1}^{N_{a}(r)} N_{w}(t ; a)} \sum_{a=1}^{N_{a}(r)} \sum_{i=1}^{N_{w}(t ; a)} \ln \left[\frac{P\left(\vec{\kappa}^{(i)}(t) \mid \text { phob }\right)}{P\left(\vec{\kappa}^{(i)}(t) \mid \mathrm{bulk}\right)}\right]
$$

and $\left\langle\lambda_{\text {phob }}\right\rangle_{0}$ is the ensemble average sampled from the ideal hydrophobic reference system (see below). The summation over $N_{a}(r)$ involves all atoms in residue $r$ and the summation over $N_{w}(t ; a)$ includes all water molecules within a cut-off of $6 \AA$ of atom $a$ at time $t .{ }^{56}$ The 
vector $\vec{\kappa}^{(i)}(t)$ describes the orientation (see above) of the $i$ th water molecule in the sampled population.

The distribution $P\left(\vec{\kappa}^{(i)}(t) \mid\right.$ phob) is determined for a reference hydrophobic reference system ('phob'), whereas $P\left(\vec{\kappa}^{(i)}(t) \mid\right.$ bulk) is determined from the actual simulations ('bulk'). ${ }^{54}$ As the quantity LH includes both, the distance $a$ of the water molecules from the interface and the orientation of a specific water molecule $\left(\theta_{\mathrm{OH} 1}, \theta_{\mathrm{OH} 2}\right)$, LH can be considered as a generalization of the radial distribution function $g(r)$. The local hydrophobicity is a measure of the statistical similarity of the sampled configurations to that of an ideal hydrophobic reference system. When sampled configurations $P\left(\vec{\kappa}^{(i)}(t) \mid\right.$ bulk) are dissimilar to the hydrophobic reference system, this indicates that the site $r$ considered is less hydrophobic, i.e. rather hydrophilic and vice versa. In other words, $\delta \lambda_{\text {phob }}^{(r)}(t) \approx 0$ for a hydrophobic environment around residue $\mathrm{r}$, whereas $\delta \lambda_{\text {phob }}^{(r)}(t)$ significantly larger than zero, the environment is hydrophilic. ${ }^{54-56}$ In previous work, ${ }^{56}$ sustained values of $\delta \lambda_{\text {phob }}^{(r)}>0.5$ were considered indicative of hydrophilicity. The magnitude of such a cutoff may, however, be somewhat system-dependent.

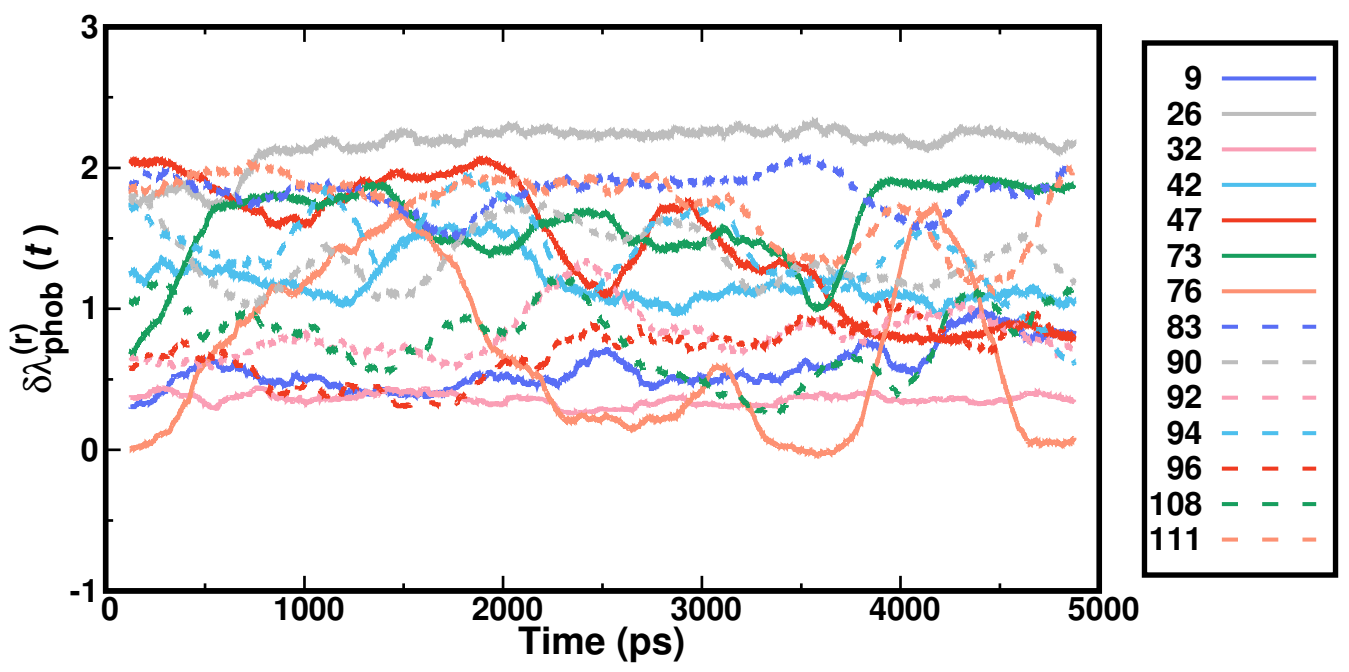

Figure 9: Local hydrophobicity as a function of time for all alanine residues from the simulation of WT Lysozyme. The LH coefficient was determined from Eq. 7. Values of $\delta \lambda_{\text {phob }} \approx 0$ indicate a hydrophobic environment of the site considered ${ }^{55,56}$ whereas values around 2 point towards a hydrophilic site. 
Figure S8 gives an overview of the average LH per residue and the fluctuations around the average for WT Lysozyme. The Alanine residues (in red) are found to include both, low and high values for LH, representative of more hydrophobic and hydrophilic environments, respectively. The change in LH as a function of simulation time (over 2 ns) for WT (blue) and $\mathrm{N}_{3}^{-}$labelled (red) Lysozyme for Ala76 is reported in Figure S9. Without spectroscopic label the Ala-residue is rather hydrophilic on average whereas with the label attached it is more hydrophobic (less hydrophilic). On the other hand, the LH can have a rather pronounced time-dependence, see Figure 9 (solid orange line for Ala76) from the 5 ns simulation of WT Lysozyme. Thus, attaching the $\mathrm{N}_{3}^{-}$label to Ala may modulate recruitment or displacement of solvent molecules.

\section{Discussion and Conclusion}

The present findings confirm that azide attached to alanine residues in Lysozyme is a structurally minimally invasive, specific infrared label to quantitatively probe the local dynamics around the modification site. This has already been reported for the PDZ2 domain. ${ }^{17}$ Similar to the situation in insulin monomer and dimer, for which the amide-I vibration was found ${ }^{41,57}$ to cover a range of $\sim 20 \mathrm{~cm}^{-1}$, attaching azide to give $\mathrm{AlaN}_{3}$ spans a comparable frequency range but in a region of the infrared spectrum (around $2100 \mathrm{~cm}^{-1}$ ) that is typically "empty". Together with their minimal impact on the overall protein structure (see Figure 3), and the still favourable extinction coefficient, ${ }^{16}$ such modifications bear great potential to resolve the structural dynamics of proteins and protein-ligand complexes at a molecular level. Studies that provide structural and spectroscopic information at the same time are of great interest for characterizing potential ligand-binding sites and for functional studies of protein allostery. 

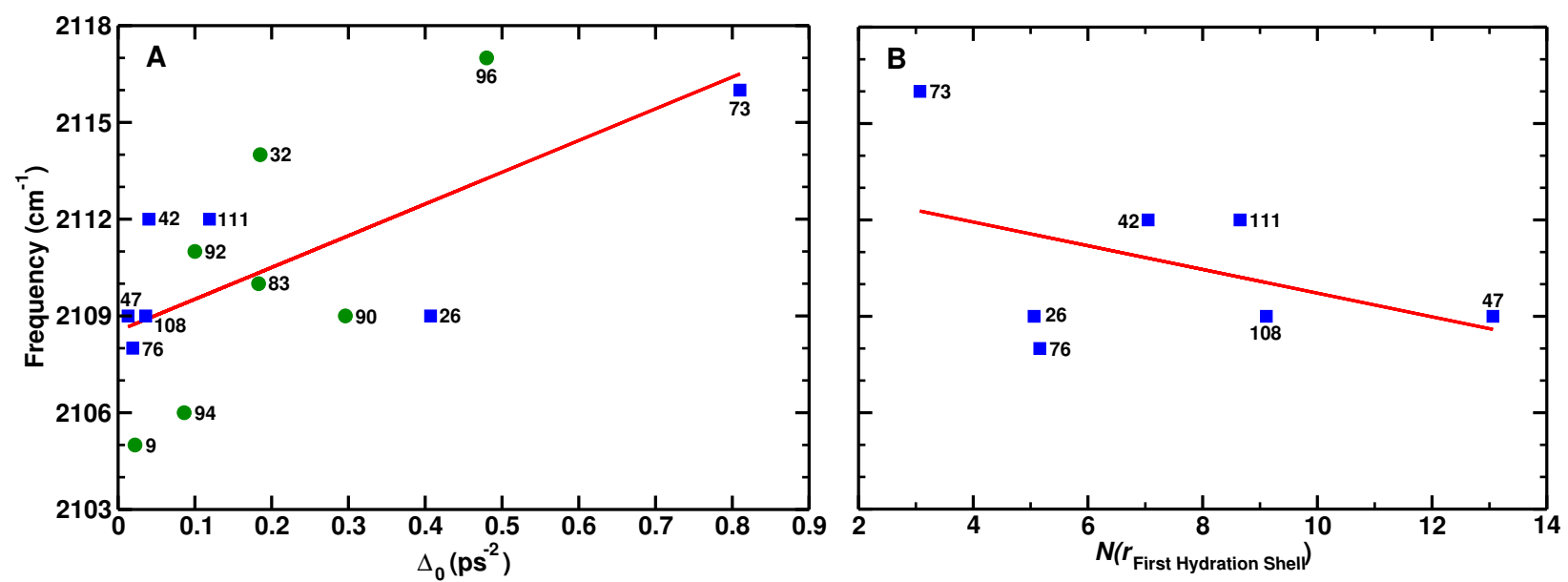

Figure 10: Correlation between the maximum of the 1-d lineshape from INM and he static offset $\Delta_{0}$ of the FFCF (panel A) and the maximum of the 1-d lineshape from INM and the number of water molecules in the first hydration shell (panel B) for the residues that has been considered to be rather "water exposed" (Set1). Residues of Set1 are shown as blue squares and those of Set2 as green circles. The solid line is an empirical linear fit and suggests that, typically, for more blue shifted frequency maxima the static component increases while the number of water molecules in the first hydration shell decreases, i.e. with increasing hydration, $\omega_{\max }$ shifts typically to the red for alanine residues in Set1.

It is of interest to delineate whether correlations can be found between structural and spectroscopic characteristics analyzed in the present work. As the dynamics is coupled and involves a potentially complicated superposition of different structural substates, no "simple" or "obvious" correlations are expected. Rather and at best, discovering trends can be expected from such an analysis. One example is shown in Figure 10B which reports the relationship between the number of water molecules in the first hydration shell (see also Figure 7) and the position of the frequency maximum $\omega_{\max }$ from the 1-d lineshape determined from the instantaneous normal mode analysis. Typically, with increasing hydration, the position of $\omega_{\max }$ shifts to the red. Similarly, the magnitude of the static offset $\Delta_{0}$ of the FFCF is related to $\omega_{\max }$ in that larger values of $\Delta_{0}$ are associated with a blue shift of the position of the frequency maximum, see Figure 10A.

Spectroscopic probes to characterize the local environment of a protein provide valuable 
information about local hydration. This is of particular relevance given the findings that individual water molecules can play decisive roles in protein function. For example, in HIV-I protease $^{58,59}$ a single catalytic water molecule was located in the active site of the protein or for insulin ${ }^{57,60}$ individual water molecules were found to attack the dimerization interface to reduce the thermodynamic stability of the dimer by a factor of two. Similarly, water molecules have been reported to play essential roles in protein folding, ${ }^{61}$ and for function. ${ }^{62,63}$ Thus, probing and characterizing the local solvent environment of particular regions of a protein can provide important insights into functional aspects of proteins.

The utility of infrared spectroscopy to study the strength of protein-ligand complexes has been proposed ${ }^{20}$ and explicitly demonstrated from molecular dynamics simulations for cyanosubstituted benzene in lysozyme. ${ }^{21}$ Using AHA as a probe, it was reported that unbound and ligand-bound PDZ2 differ in that the frequency correlation function for the two systems decay to different levels at longer correlation times. Similarly, infrared spectroscopy is also a sensitive probe - both, in terms of spectroscopy and dynamics - to characterize proteinprotein interactions. ${ }^{41}$ Together with experimental studies, ${ }^{64-66}$ such efforts pave the way for functional, in vivo studies of protein-ligand and protein-protein association. ${ }^{67}$

In conclusion, the present work provides a comprehensive analysis of the spectroscopy and dynamics of azide-labelled alanine in Lysozyme. The results demonstrate that $\mathrm{AlaN}_{3}$ is a positionally sensitive probe for the local dynamics, covering a frequency range of $\sim 15 \mathrm{~cm}^{-1}$. This is consistent with findings from selective replacements of amino acids in PDZ2 which reported a frequency span of $\sim 10 \mathrm{~cm}^{-1}$ for replacements of Val, Ala, or Glu by AHA. ${ }^{16}$ Furthermore, the long-time decay constants $\tau_{2}$ range from $\sim 1$ to $\sim 10$ ps which compares with experimentally measured correlation times of 3 ps. ${ }^{16}$ Attaching azide to alanine residues can yield dynamics that decays to zero on the few ps time scale (i.e. $\Delta_{0} \sim 0 \mathrm{ps}^{-1}$ ) or to a remaining inhomogeneous contribution of $\sim 0.5 \mathrm{ps}^{-1}$ (corresponding to $2.5 \mathrm{~cm}^{-1}$ ). One 
exciting prospect of this is to determine how the spectroscopy and dynamics of the modification site changes upon ligand binding to the active site for Lysozyme or other proteins.

\section{Acknowledgments}

The authors gratefully acknowledge financial support from the Swiss National Science Foundation through grant 200021-117810 and to the NCCR-MUST. The authors thank Prof. P. Hamm and Dr. D. Koner for discussions on the experiments and some of the electronic structure calculations.

\section{Data Availability Statement}

The data that support the findings of this study are available from the corresponding author upon reasonable request. 


\section{References}

(1) Plitzko, J. M.; Schuler, B.; Selenko, P. Structural Biology outside the box - inside the cell. Curr. Op. Struct. Biol. 2017, 46, 110-121.

(2) Guo, J.; Zhou, H.-X. Protein Allostery and Conformational Dynamics. Chem. Rev. 2016, 116, 6503-6515.

(3) Lu, S.; He, X.; Ni, D.; Zhang, J. Allosteric Modulator Discovery: From Serendipity to Structure-Based Design. J. Med. Chem. 2019, 62, 6405-6421.

(4) Hamm, P.; Zanni, M. Concepts and Methods of 2D Infrared Spectroscopy; Cambridge University Press: New York, 2011.

(5) Waegele, M. M.; Culik, R. M.; Gai, F. Site-Specific Spectroscopic Reporters of the Local Electric Field, Hydration, Structure, and Dynamics of Biomolecules. J. Phys. Chem. Lett. 2011, 2, 2598-2609.

(6) Koziol, K. L.; Johnson, P. J. M.; Stucki-Buchli, B.; Waldauer, S. A.; Hamm, P. Fast infrared spectroscopy of protein dynamics: advancing sensitivity and selectivity. Curr. Op. Struct. Biol. 2015, 34, 1-6.

(7) Horness, R. E.; Basom, E. J.; Thielges, M. C. Site-selective characterization of Src homology 3 domain molecular recognition with cyanophenylalanine infrared probes. Anal. Chem. 2015, 7, 7234-7241.

(8) Getahun, Z.; Huang, C.; Wang, T.; De Leon, B.; DeGrado, W.; Gai, F. Using nitrilederivatized amino acids as infrared probes of local environment. J. Am. Chem. Soc. 2003, 125, 405-411.

(9) Kozinski, M.; Garrett-Roe, S.; Hamm, P. 2D-IR spectroscopy of the sulfhydryl band of cysteines in the hydrophobic core of proteins. J. Phys. Chem. B 2008, 112, 7645-7650. 
(10) Zimmermann, J.; Thielges, M. C.; Yu, W.; Dawson, P. E.; Romesberg, F. E. CarbonDeuterium Bonds as Site-Specific and Nonperturbative Probes for Time-Resolved Studies of Protein Dynamics and Folding. J. Phys. Chem. Lett. 2011, 2, 412-416.

(11) Woys, A. M.; Mukherjee, S. S.; Skoff, D. R.; Moran, S. D.; Zanni, M. T. A Strongly Absorbing Class of Non-Natural Labels for Probing Protein Electrostatics and Solvation with FTIR and 2D IR Spectroscopies. J. Phys. Chem. B 2013, 117, 5009-5018.

(12) Bagchi, S.; Boxer, S. G.; Fayer, M. D. Ribonuclease S Dynamics Measured Using a Nitrile Label with 2D IR Vibrational Echo Spectroscopy. J. Phys. Chem. B 2012, 116, $4034-4042$.

(13) Zimmermann, J.; Thielges, M. C.; Seo, Y. J.; Dawson, P. E.; Romesberg, F. E. Cyano Groups as Probes of Protein Microenvironments and Dynamics. Angew. Chem. Int. Ed. 2011, 50, 8333-8337.

(14) van Wilderen, L. J. G. W.; Kern-Michler, D.; Mueller-Werkmeister, H. M.; Bredenbeck, J. Vibrational dynamics and solvatochromism of the label SCN in various solvents and hemoglobin by time dependent IR and 2D-IR spectroscopy. Phys. Chem. Chem. Phys. 2014, 16, 19643-19653.

(15) Lee, G.; Kossowska, D.; Lim, J.; Kim, S.; Han, H.; Kwak, K.; Cho, M. Cyanamide as an Infrared Reporter: Comparison of Vibrational Properties between Nitriles Bonded to N and C Atoms. J. Phys. Chem. B 2018, 122, 4035-4044.

(16) Bloem, R.; Koziol, K.; Waldauer, S. A.; Buchli, B.; Walser, R.; Samatanga, B.; Jelesarov, I.; Hamm, P. Ligand Binding Studied by 2D IR Spectroscopy Using the Azidohomoalanine Label. J. Phys. Chem. B 2012, 116, 13705-13712.

(17) Zanobini, C.; Bozovic, O.; Jankovic, B.; Koziol, K. L.; Johnson, P. J. M.; Hamm, P.; Gulzar, A.; Wolf, S.; Stock, G. Azidohomoalanine: A Minimally Invasive, Versatile, 
and Sensitive Infrared Label in Proteins To Study Ligand Binding. J. Phys. Chem. B 2018, 122, 10118-10125.

(18) Kiick, K.; Saxon, E.; Tirrell, D.; Bertozzi, C. Incorporation of azides into recombinant proteins for chemoselective modification by the Staudinger ligation. Proc. Natl. Acad. Sci. 2002, 99, 19-24.

(19) Koziol, K. L.; Johnson, P. J. M.; Stucki-Buchli, B.; Waldauer, S. A.; Hamm, P. Fast Infrared Spectroscopy of Protein Dynamics: Advancing Sensitivity and Selectivity. Curr. Op. Struct. Biol. 2015, 34, 1-6.

(20) Suydam, I. T.; Snow, C. D.; Pande, V. S.; Boxer, S. G. Electric Fields at the Active Site of an Enzyme : Direct Comparison of Experiment with Theory. Science 2006, 313, 200-204.

(21) Mondal, P.; Meuwly, M. Vibrational Stark Spectroscopy for Assessing Ligand-Binding Strengths in a Protein. Phys. Chem. Chem. Phys. 2017, 19, 16131-16143.

(22) Salehi, S. M.; Koner, D.; Meuwly, M. Vibrational Spectroscopy of $\mathrm{N}_{3}^{-}$in the Gas and Condensed Phase. J. Phys. Chem. B 2019, 123, 3282-3290.

(23) Ho, T.-S.; Rabitz, R. A General Method for Constructing Multidimensional Molecular Potential Energy Surfaces from ab Initio Calculations. J. Chem. Phys. 1996, 104, $2584-2597$.

(24) Unke, O. T.; Meuwly, M. Toolkit for the Construction of Reproducing Kernel-Based Representations of Data: Application to Multidimensional Potential Energy Surfaces. J. Chem. Inf. Model. 2017, 5\%, 1923-1931.

(25) Brooks, B. R.; Brooks III, C. L.; MacKerell Jr., A. D.; Nilsson, L.; Petrella, R. J.; Roux, B.; Won, Y.; Archontis, G.; Bartels, C.; Boresch, S. et al. CHARMM: The Biomolecular Simulation Program. J. Comp. Chem. 2009, 30, 1545-1614. 
(26) MacKerell, A. D.; Bashford, D.; Bellott, M.; Dunbrack, R. L.; Evanseck, J. D.; Field, M. J.; Fischer, S.; Gao, J.; Guo, H.; Ha, S. et al. All-atom Empirical Potential for Molecular Modeling and Dynamics Studies of Proteins. J. Phys. Chem. B 1998, 102, 3586-3616.

(27) Chiba-Kamoshida, K.; Matsui, T.; Ostermann, A.; Chatake, T.; Ohhara, T.; Tanaka, I.; Yutani, K.; Niimura, N. X-ray crystal structure of wild type human lysozyme in $\mathrm{D}_{2} \mathrm{O}$. DOI: $10.2210 /$ pdb3fe0/pdb.

(28) Jorgensen, W. L.; Chandrasekhar, J.; Madura, J. D.; Impey, R. W.; Klein, M. L. Comparison of Simple Potential Functions for Simulating Liquid Water. J. Chem. Phys. 1983, 79, 926-935.

(29) Steinbach, P. J.; Brooks, B. R. New Spherical-Cutoff Methods for Long-Range Forces in Macromolecular Simulation. J. Comput. Chem. 1994, 15, 667-683.

(30) Gunsteren, W. V.; Berendsen, H. Algorithms for Macromolecular Dynamics and Constraint Dynamics. Mol. Phys. 1997, 34, 1311-1327.

(31) Schwilk, M.; Ma, Q.; Koeppl, C.; Werner, H.-J. Scalable Electron Correlation Methods. 3. Efficient and Accurate Parallel Local Coupled Cluster with Pair Natural Orbitals (PNO-LCCSD). J. Chem. Theo. Comp. 2017, 13, 3650-3675.

(32) Ma, Q.; Schwilk, M.; Koeppl, C.; Werner, H.-J. Scalable Electron Correlation Methods. 4. Parallel Explicitly Correlated Local Coupled Cluster with Pair Natural Orbitals (PNO-LCCSD-F12) (vol 13, pg 4871, 2017). J. Chem. Theo. Comp. 2018, 14, 6750.

(33) Dunning, T. H., Jr. Gaussian basis sets for use in correlated molecular calculations. I. The atoms boron through neon and hydrogen. J. Chem. Phys. 1989, 90, 1007-1023.

(34) Werner, H.-J.; Knowles, P. J.; Knizia, G.; Manby, F. R.; Schütz, M.; Celani, P.; 
Györffy, W.; Kats, D.; Korona, T.; Lindh, R. et al. MOLPRO, Version 2012.1, A Package of ab Initio Programs. 2012.

(35) Zoete, V.; Cuendet, M.; Grosdidier, A.; Michielin, O. SwissParam: A Fast Force Field Generation Tool for Small Organic Molecules. J. Comp. Chem. 2011, 32, 2359-2368.

(36) Meuwly, M.; Hutson, J. The Potential Energy Surface and Near-dissociation States of He-H ${ }_{2}^{+}$. J. Chem. Phys. 1999, 110, 3418-3427.

(37) Soldán, P.; Hutson, J. M. On the long-range and short-range behavior of potentials from reproducing kernel Hilbert space interpolation. J. Chem. Phys. 2000, 112, 4415-4416.

(38) Hollebeek, T.; Ho, T.-S.; Rabitz, H. Constructing Multidimensional Molecular Potential Energy Surfaces from ab Initio Data. Ann. Rev. Phys. Chem. 1999, 50, 537-570.

(39) Foster, J. P.; Weinhold, F. Natural Hybrid Orbitals. J. Am. Chem. Soc. 1980, 102, $7211-18$.

(40) Frisch, M. J.; Trucks, G. W.; Schlegel, H. B.; Scuseria, G. E.; Robb, M. A.; Cheeseman, J. R.; Scalmani, G.; Barone, V.; Petersson, G. A.; Nakatsuji, H. et al. Gaussian 16 Revision C.09. 2016; Gaussian Inc. Wallingford CT.

(41) Salehi, S. M.; Koner, D.; Meuwly, M. Dynamics and Infrared Spectrocopy of Monomeric and Dimeric Wild Type and Mutant Insulin. J. Phys. Chem. B 2020, in print, in print.

(42) Maekawa, H.; Ohta, K.; Tominaga, K. Spectral Diffusion of the Anti-Symmetric Stretching Mode of Azide Ion in a Reverse Micelle Studied by Infrared Three-Pulse Photon Echo Method. Phys. Chem. Chem. Phys. 2004, 6, 4074-4077.

(43) Zhong, Q.; Baronavski, A.; Owrutsky, J. Vibrational Energy Relaxation of Aqueous Azide Ion Confined in Reverse Micelles. J. Chem. Phys. 2003, 118, 7074-7080.

(44) Moller, K.; Rey, R.; Hynes, J. Hydrogen Bond Dynamics in Water and Ultrafast Infrared Spectroscopy: A Theoretical Study. J. Phys. Chem. A 2004, 108, 1275-1289. 
(45) Virtanen, P.; Gommers, R.; Oliphant, T. E.; Haberland, M.; Reddy, T.; Cournapeau, D.; Burovski, E.; Peterson, P.; Weckesser, W.; Bright, J. et al. SciPy 1.0: Fundamental Algorithms for Scientific Computing in Python. Nature Methods 2020, 17, 261-272.

(46) Okuda, M.; Ohta, K.; Tominaga, K. Vibrational dynamics of azide-derivatized amino acids studied by nonlinear infrared spectroscopy. jcp 2015, 142 .

(47) Bowman, J. M.; Gazdy, B. A simple method to adjust potential energy surfaces: Application to HCO. J. Chem. Phys. 1991, 94, 816-817.

(48) Meuwly, M.; Hutson, J. M. Morphing ab Initio potentials: A systematic study of $\mathrm{Ne}^{-}$ HF. J. Chem. Phys. 1999, 110, 8338-8347.

(49) Li, S.; Schmidt, J. R.; Piryatinski, A.; Lawrence, C. P.; Skinner, J. L. Vibrational Spectral Diffusion of Azide in Water. J. Phys. Chem. B 2006, 110, 18933-18938.

(50) Lee, M. W.; Carr, J. K.; Göllner, M.; Hamm, P.; Meuwly, M. 2D IR Spectra of Cyanide in Water Investigated by Molecular Dynamics Simulations. J. Chem. Phys. 2013, 139, 054506 .

(51) Hamm, P.; Lim, M.; Hochstrasser, R. M. Structure of the Amide I Band of Peptides Measured by Femtosecond Nonlinear-Infrared Spectroscopy. J. Phys. Chem. B 1998, $5647,6123-6138$.

(52) Cazade, P.-A.; Bereau, T.; Meuwly, M. Computational Two-Dimensional Infrared Spectroscopy without Maps: N-Methylacetamide in Water. J. Phys. Chem. B 2014, 118, 8135-8147.

(53) Koner, D.; Salehi, S. M.; Mondal, P.; Meuwly, M. Non-conventional force fields for applications in spectroscopy and chemical reaction dynamics. J. Chem. Phys. 2020, 153, 010901. 
(54) Shin, S.; Willard, A. P. Characterizing Hydration Properties Based on the Orientational Structure of Interfacial Water Molecules. J. Chem. Theo. Comp. 2018, 14, 461-465.

(55) Shin, S.; Willard, A. P. Water's Interfacial Hydrogen Bonding Structure Reveals the Effective Strength of Surface-Water Interactions. J. Phys. Chem. B 2018, 122, 67816789.

(56) Pezzella, M.; El Hage, K.; Niesen, M. J.; Shin, S.; Willard, A. P.; Meuwly, M.; Karplus, M. Water dynamics around proteins: T-and R-States of hemoglobin and melittin. J. Phys. Chem. B 2020, 124, 6540-6554.

(57) Desmond, J. L.; Koner, D.; Meuwly, M. Probing the Differential Dynamics of the Monomeric and Dimeric Insulin from Amide-I IR Spectroscopy. J. Phys. Chem. B 2019, 123, 6588-6598.

(58) Baldwin, E. T.; Bhat, T. N.; Gulnik, S.; Liu, B.; Topol, I. A.; Kiso, Y.; Mimoto, T.; Mitsuya, H.; Erickson, J. W. Structure of HIV-1 protease with KNI-272, a tight-binding transition-state analog containing allophenylnorstatine. Structure 1995, 3, 581-590.

(59) Prashar, V.; Bihani, S.; Das, A.; Ferrer, J.-L.; Hosur, M. Catalytic water co-existing with a product peptide in the active site of HIV-1 protease revealed by X-ray structure analysis. PloS one 2009, 4, e7860.

(60) Raghunathan, S.; El Hage, K.; Desmond, J. L.; Zhang, L.; Meuwly, M. The Role of Water in the Stability of Wild-type and Mutant Insulin Dimers. J. Phys. Chem. B 2018, 122, 7038-7048.

(61) Schiro, G.; Fichou, Y.; Gallat, F.-X.; Wood, K.; Gabel, F.; Moulin, M.; Haertlein, M.; Heyden, M.; Colletier, J.-P.; Orecchini, A. et al. Translational diffusion of hydration water correlates with functional motions in folded and intrinsically disordered proteins. Nuovo Cim. 2015, 6, 1-8. 
(62) Pocker, Y. Water in enzyme reactions: biophysical aspects of hydration-dehydration processes. Cell. Mol. Life Sci. 2000, 57, 1008-1017.

(63) Pal, S.; Zewail, A. Dynamics of water in biological recognition. Chem. Rev. 2004, 104, 2099-2123.

(64) Zhang, X.-X.; Jones, K. C.; Fitzpatrick, A.; Peng, C. S.; Feng, C.-J.; Baiz, C. R.; Tokmakoff, A. Studying Protein-Protein Binding through T-Jump Induced Dissociation: Transient 2D IR Spectroscopy of Insulin Dimer. J. Phys. Chem. B 2016, 120, $5134-5145$.

(65) Zhang, X.-X.; Tokmakoff, A. Revealing the Dynamical Role of Co-solvents in the Coupled Folding and Dimerization of Insulin. J. Phys. Chem. Lett. 2020, 11, 4353-4358.

(66) Antoszewski, A.; Feng, C.-J.; Vani, B. P.; Thiede, E. H.; Hong, L.; Weare, J.; Tokmakoff, A.; Dinner, A. R. Insulin Dissociates by Diverse Mechanisms of Coupled Unfolding and Unbinding. J. Phys. Chem. B 2020, 124, 5571-5587.

(67) Miller, L. M.; Bourassa, M. W.; Smith, R. J. FTIR spectroscopic imaging of protein aggregation in living cells. Bioch. Bioph. Acta 2013, 1828, 2339-2346. 


\title{
Supporting Information: Site-Selected Dynamics of Azidolysozyme
}

\author{
Seyedeh Maryam Salehi and Markus Meuwly* \\ Department of Chemistry, University of Basel, Klingelbergstrasse 80, CH-4056 Basel, \\ Switzerland. \\ E-mail: m.meuwly@unibas.ch
}

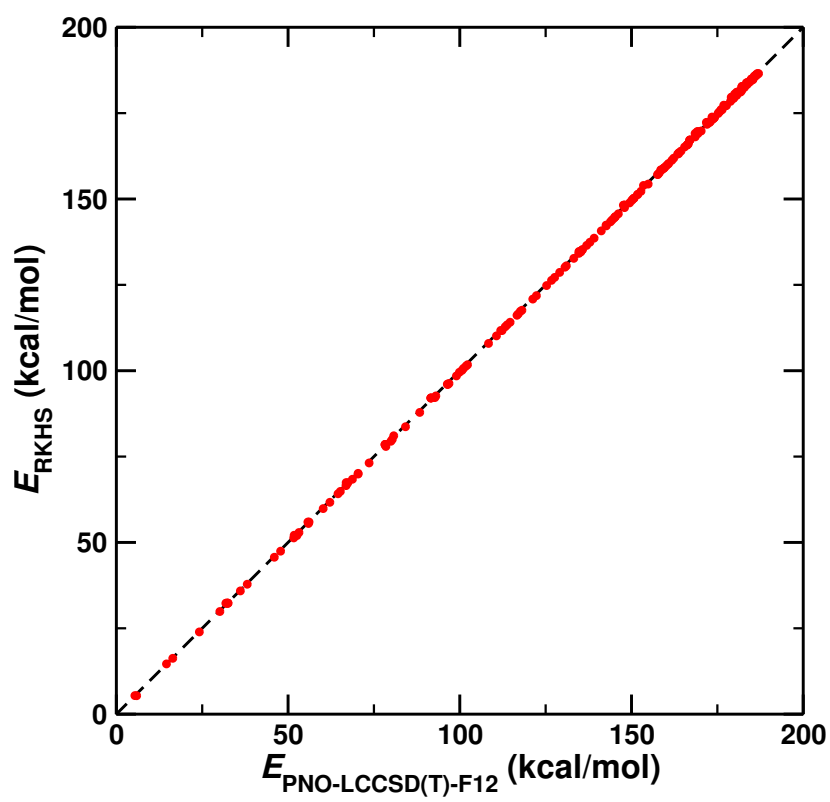

Figure S1: Correlation between ab initio and RKHS interpolation for 230 randomly selected geometries. The $R^{2}=0.9999$ and the RMSD is $0.38 \mathrm{kcal} / \mathrm{mol}$ over a range of $180 \mathrm{kcal} / \mathrm{mol}$. 


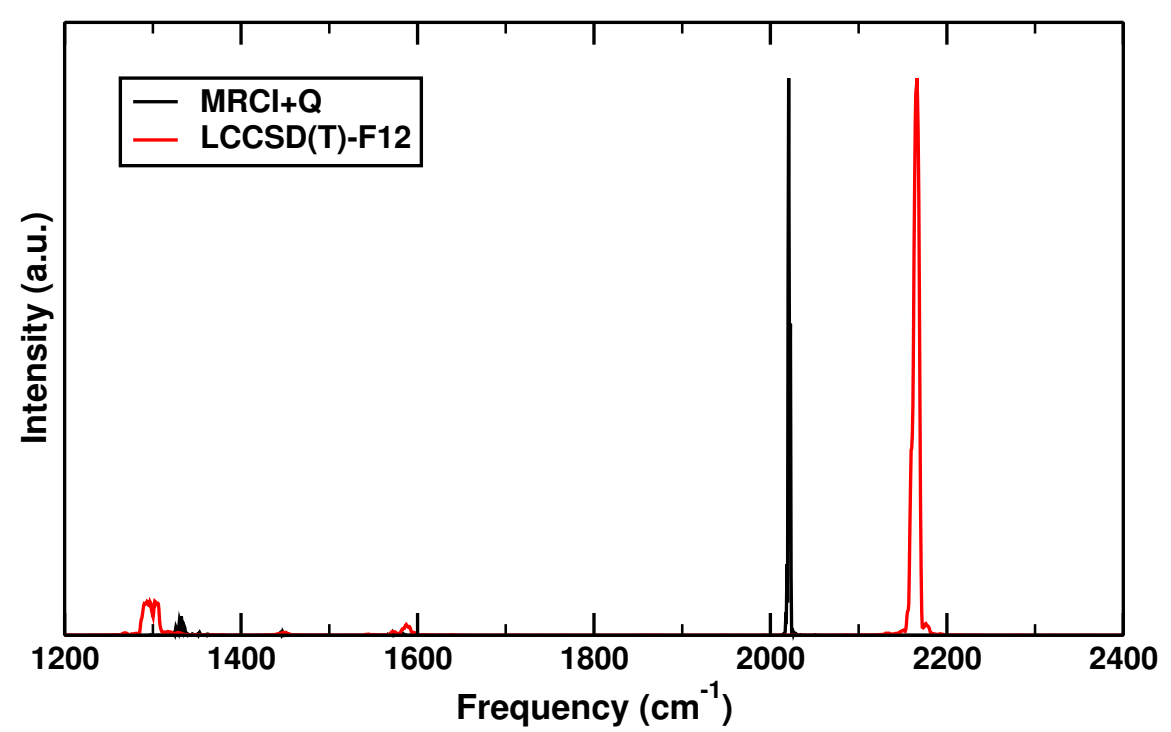

Figure S2: Power spectrum based on the N1-N2 separation for AHA in the gas phase and from MRCI+Q and LCCSD(T)-F12 surface.

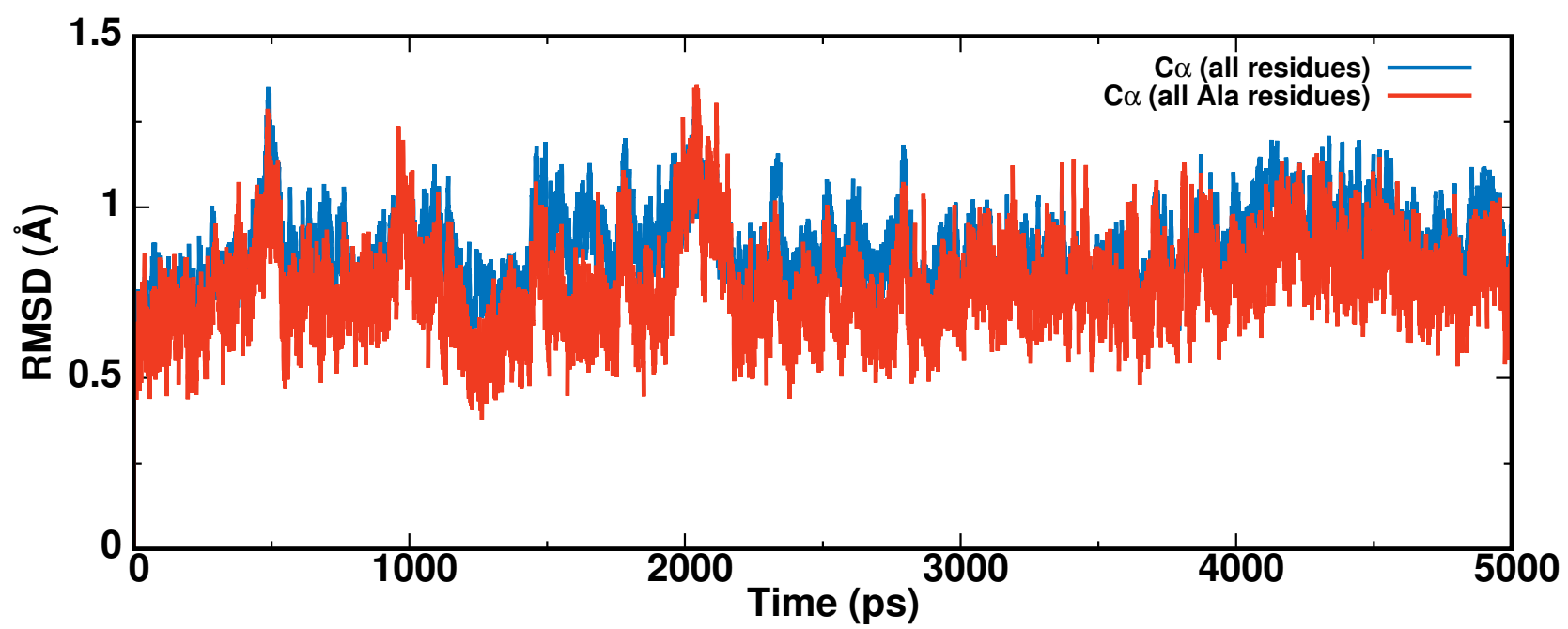

Figure S3: The structural RMSD for the $\mathrm{C}_{\alpha}$ atoms from all residues (blue) and for the 14 Ala residues (red) for WT Lysozyme. 


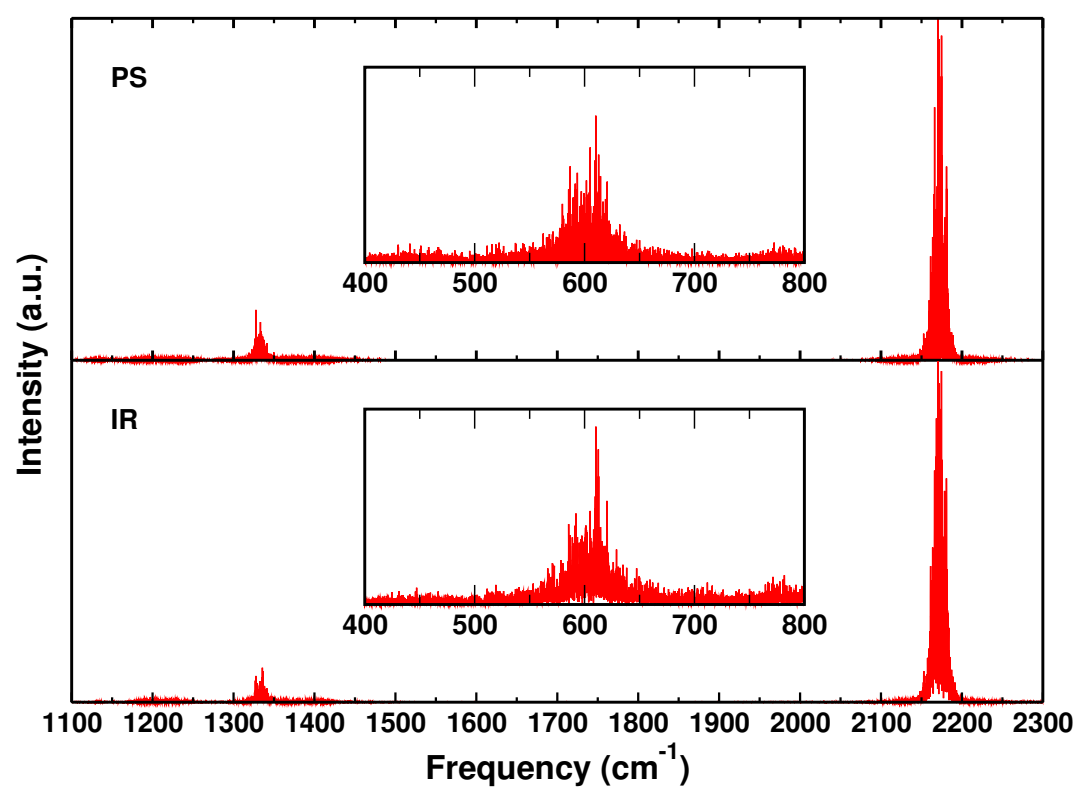

Figure S4: Power (PS, top panel) and IR (bottom panel) spectrum for Ala $47 \mathrm{~N}_{3}$. The power spectrum is based on N2-N3 bond displacement. The inset shows the bending mode of the Azide group. IR spectrum is calculated the Fourier transform of the molecular dipole moment autocorrelation function. 


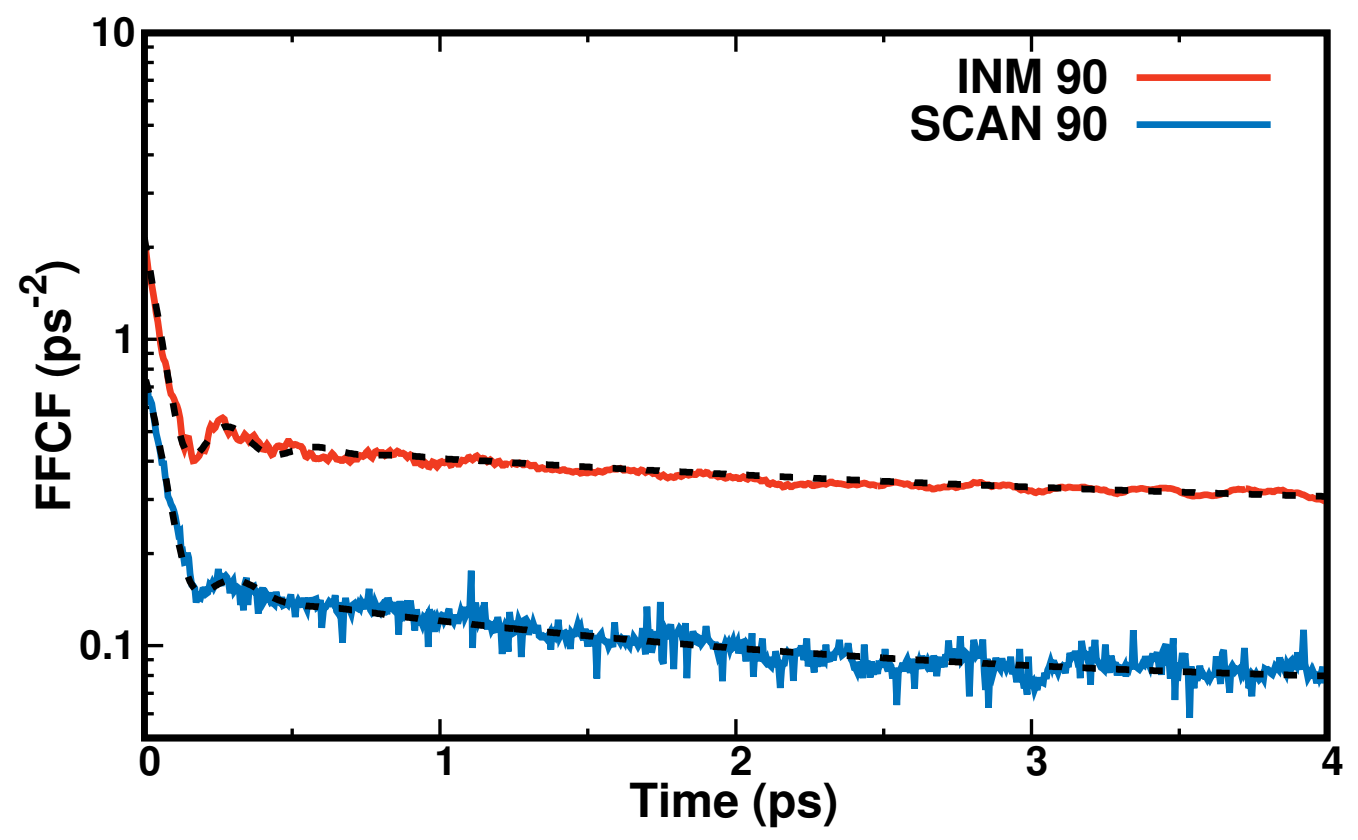

Figure S5: The FFCF for Ala90 based on INM (red) and scan (blue) frequencies. The dashed lines show the corresponding fit to Eq. ?? with 3 time scales and the fitting parameters are as follows: INM: $a_{1}=0.29, \gamma=21.00, \tau_{1}=0.18 \mathrm{ps}, a_{2}=1.38, \tau_{2}=0.05 \mathrm{ps}, a_{3}=0.22$, $\tau_{3}=2.70 \mathrm{ps}, \Delta_{0}=0.25$ and scan: $a_{1}=0.21, \gamma=17.50, \tau_{1}=0.11 \mathrm{ps}, a_{2}=0.39, \tau_{2}=0.08$ ps, $a_{3}=0.09, \tau_{3}=1.62 \mathrm{ps}, \Delta_{0}=0.07$. The comparison shows that the two different ways to determine the instantaneous frequency $(\omega(t)$ and $\nu(t)$, respectively) does not affect the overall appearance of the FFCF except for the magnitude of the asymptotic value $\Delta_{0}$. 


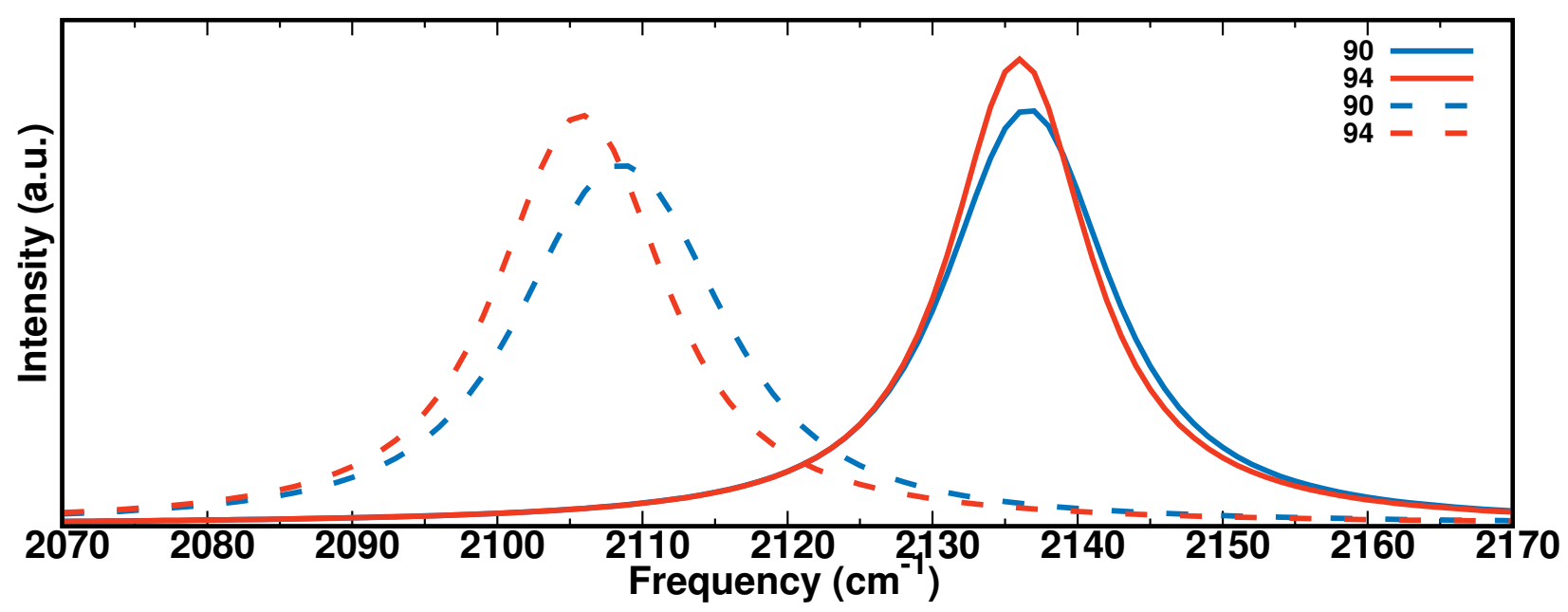

Figure S6: 1D IR spectra for Ala90N 3 and Ala94N 3 of lysozyme obtained from frequency calculations using "scan" (solid lines) and INM (dashed lines). Both analyses agree in that the frequency maximum for $\mathrm{Ala}_{90 \mathrm{~N}_{3}}$ is to the blue of that for Ala94N 3 but the magnitude of the shift differs for the two approaches.

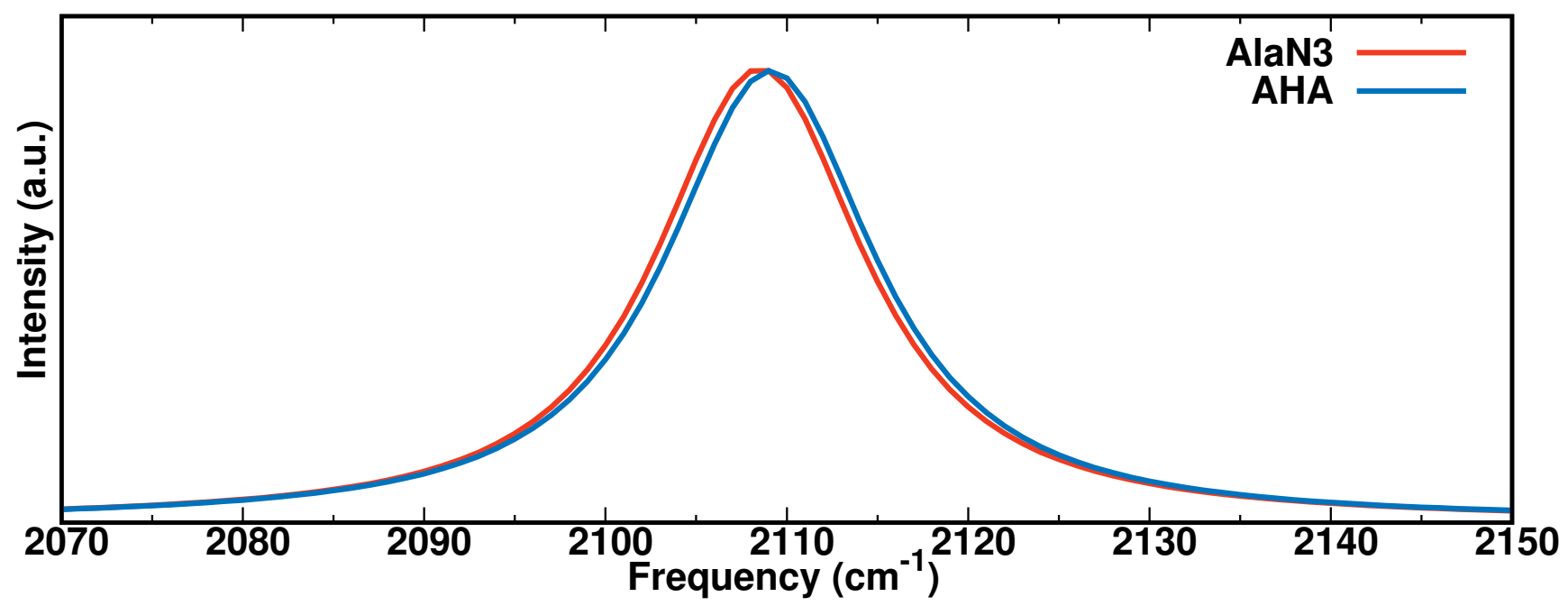

Figure S7: Comparison between the 1D-IR spectra of $\mathrm{AlaN}_{3}$ and AHA at position Ala47 in Lysozyme. For AHA, an additional $\mathrm{CH}_{2}$ group is inserted before the $\mathrm{N}_{3}^{-}$label. The lineshapes for azidoalanine and azidohomoalanine are very similar. The position of the frequency maximum for the asymmetric stretch of the azide label differs by less than 1 $\mathrm{cm}^{-1}$. 


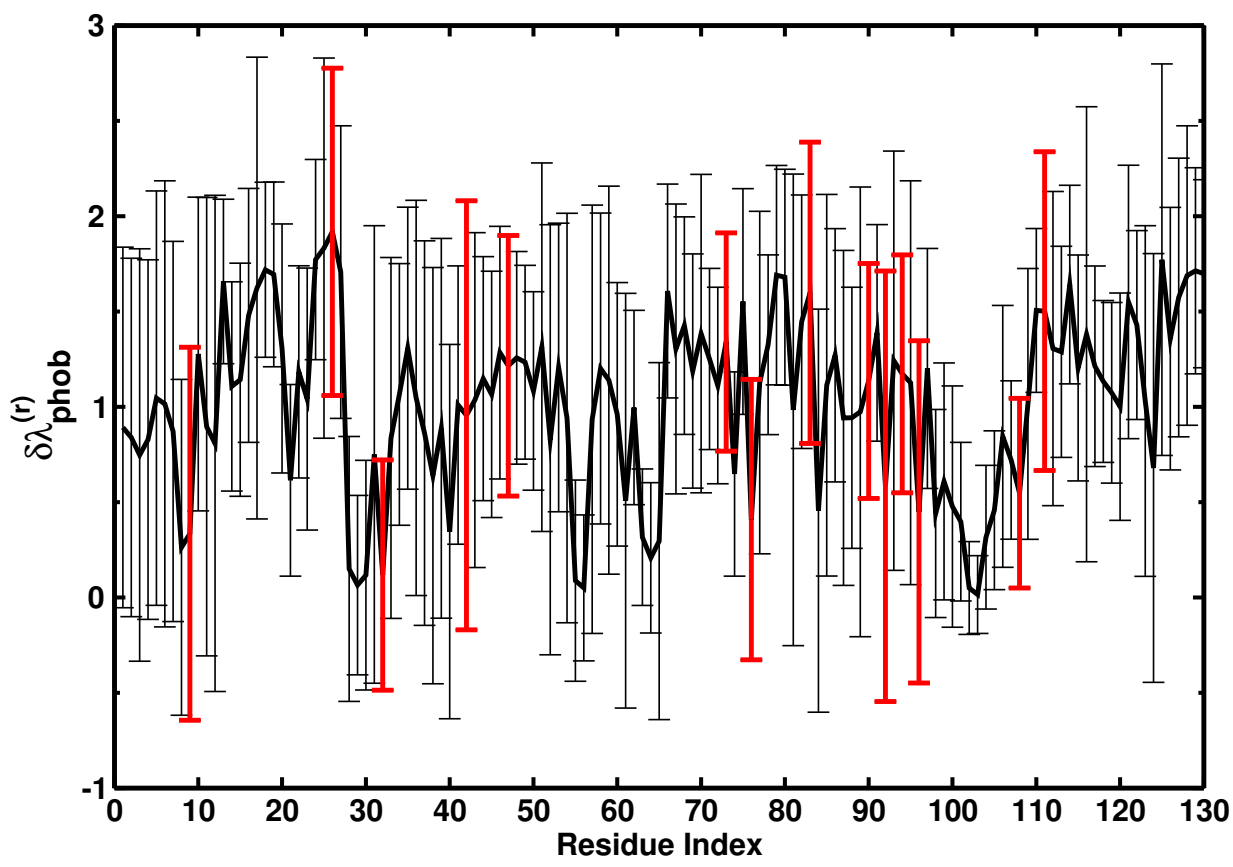

Figure S8: Average local hydrophobicity together with fluctuations for WT Lysozyme (no $\mathrm{N}_{3}^{-}$labels attached) for the $5 \mathrm{~ns}$ simulation. LH for all residues in black and for Ala in red.

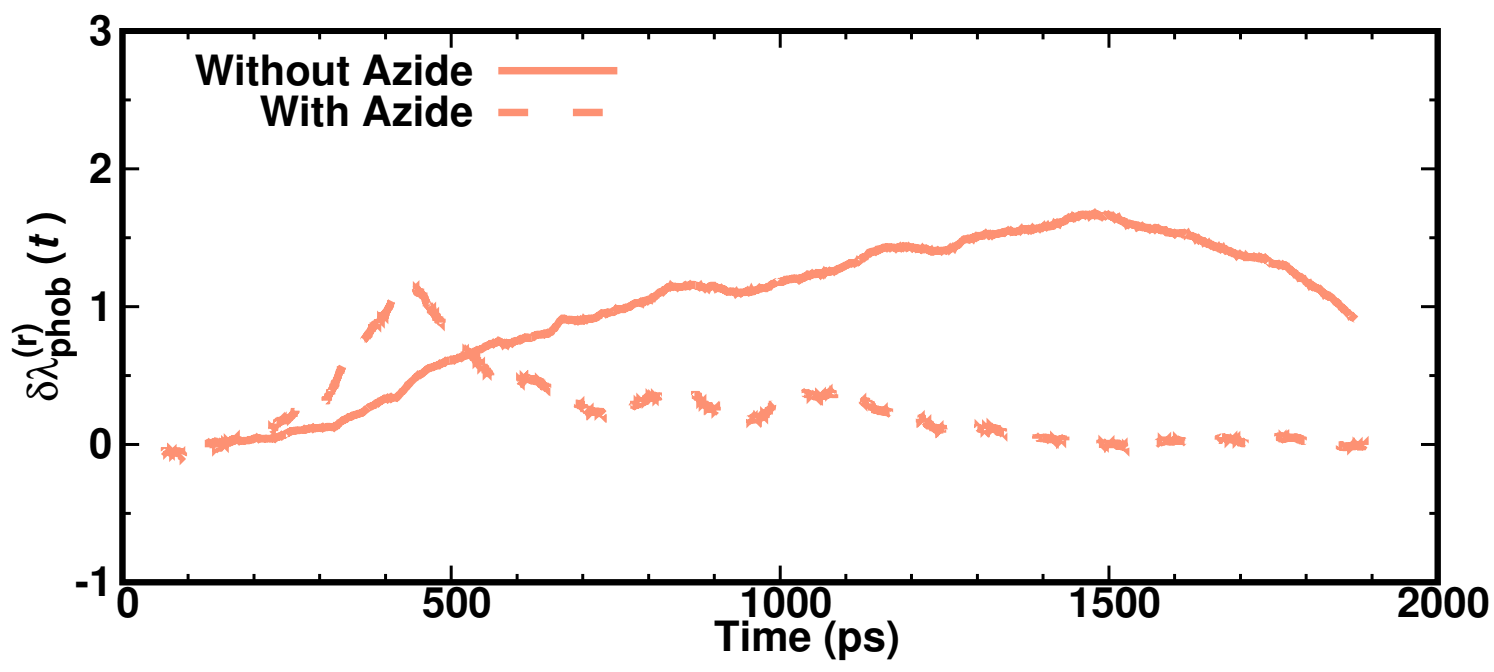

Figure S9: Local hydrophobicity for residue 76 with and without azide group attached to Ala for 2 ns. The effect of attaching azide on the LH, and hence the hydration itself, is clearly visible. 